Publ. RIMS, Kyoto Univ.

15 (1979), 1-45

\title{
Surjectivité dans les Espaces en Dualité
}

\author{
Par \\ Eliahou ZINI*
}

\section{Introduction}

Depuis plusieurs années le probléme de la surjectivité d'opérateurs non linéaires dans les espaces non réflexifs se posait. Depuis les travaux de Brezis [1] on savait déjà résoudre certains de ces problèmes, en particulier dans le cas où les opérateurs étaient définis sur un espace de Banach dual à valeurs dans son prédual. Depuis un grand pas en avant a été franchi avec les résultats de Gossez [11] [12] [13], Donaldson [6], Fougeres [10], Robert [21] [22]. Cependant de nombreux problèmes sont restés ouverts.

Nous nous sommes proposés ici, essentiellement deux buts. Le premier, de donner des résultats, englobant ceux des travaux précédents, mais surtout permettant de résoudre certains nouveaux problèmes. Le second, de mettre en évidence le fait que la plupart des théories variationnelles utilisées dans les travaux ci-dessus côtés, ainsi que celles des travaux de Brezis [2], Browder [3], Browder-Hess [4], Hess [14] [17], Bui An Ton [23] [24], Lions [20],... n'étaient toutes qu'une forme particulière de certains résultats généraux, et pouvaient se regrouper tous dans une même théorie générale.

Nous montrerons en particulier, comment en jouant sur des topologies, différentes en général des topologies naturelles, on peut éliminer tous un ensemble de restrictions émises en général, comme la reflexité, la séparabilité, espace de Banach dual, utilisation de filtres.

Pour des raisons de longueur, le cas d'opérateurs à domaine quelconque n'a été qu'effleuré ici sur chapitre 3 paragraphe 3 . Des résultats, non des plus généraux, pourront être trouvés dans [28], [29].

Communiqué par S. Matsuura, mai le 8, 1976.

* Département de Mathématique, Weizmann Institut, Rehovot, Israel.

Present address: Dept. of Math., I.I.T. Technion Haifa Israel. 


\section{Chapitre 1. Définitions et Proprietes de Base des Opérateurs}

Comme le nom du chapitre l'indique, notre but, dans un premier stade est de définir l'ensemble des opérateurs mis en jeu dans ce travail. Dans un second stade, nous essaierons de matter à jour certains rapports entre ces opérateurs. En particulier nous montrerons que sous des conditions assez faibles, la somme de deux opérateurs de type pseudomonotone (comme nous les définirons dans la suite) reste de type pseudomonotone.

Nous introduisons quatre espaces localement convexes $E, E_{0}, F, F_{0}$ avec certains liens bien précis entre ces espaces. Nous nous referrerons alors à des opérateurs en général multivalents à domaine inclus dans $E$ et à valeurs dans $F_{0}$. Nous subdiviserons ces opérateurs en deux catégories. Une première catégorie incluant les opérateurs dont la définition introduit des suites généralisées, et une seconde dont la définition n'utilise que la notion de suite ordinaire. On peut préciser dès maintenant, que ce partage sera intrinsèquement lié au choix des topologies sur les espaces. Dans la mesure où ces topologies vérifient certaines conditions particulières, il suffira, afin de résoudre certaines équations de se servir d'opératurs liés à des suites ordinaires.

Signalons ici, que les opérateurs que nous introduirons, incluent comme cas particulier tous les opérateurs de type $(M),(G P)$ ou $(P)$ introduits par $H$. Brezis [1] ou Browder-Hess [4], Hess [14],... Gossez [12], [13] Donaldson [6] Bui An Ton [23],... et ainsi tous les théorèmes que nous obtiendrons en ce qui concernent les rapports entre ces opérateurs, généralisent automatiquement, les résultats correspondants de ces articles.

\section{1. Notations}

Soit $A$ un ensemble et $\mathscr{I}_{A}$ une topologie sur $A$. On désignera par $\left(A, \mathscr{I}_{A}\right)$ l'espace topologique défini par l'ensemble $A$ muni de la topologie $\mathscr{I}_{A}$. Soit $B$ un sous-ensemble de $A$, on désignera $\operatorname{par}\left(B, \mathscr{I}_{A} / B\right)$, l'espace $B$ muni de la topologie induite par $\mathscr{I}_{A}$.

Si $\left(A_{1}, \mathscr{I}_{1}\right)$ et $\left(A_{2}, \mathscr{I}_{2}\right)$ désignent deux espaces topologiques, la notation $\left(A_{1}, \mathscr{I}_{1}\right) \quad\left(A_{2}, \mathscr{I}_{2}\right)$ exprimera le fait qu'il existe une injection continue du premier espace dans le second. Si de plus $A_{1}$ et $A_{2}$ sont des 
espaces vectoriels topologiques, cette injection sera toujours supposée linéaire.

Par contre la notation $A_{1} \subseteq A_{2}$ exprimera le fait que $A_{1}$ s'identifie à un sous ensemble de $A_{2}$ (de façon indépendante de toute notion de topologie).

Dans toute la suite $(E, F)$ et $\left(E_{0}, F_{0}\right)$ désignerons deux couples d'espaces vectoriels en dualité sur $R$. Ces dualités seront notées $($,$) et$ $(,)_{0}$ respectivement.

Sur $E_{0}$ on définit deux topologies. Une première topologie d'espace normé notée \|\|$_{E_{0}}$ et une seconde topologie localement convexe $\mathscr{I}_{E_{0}}$. On notera $\left(\widehat{E}_{0}, \widehat{\mathcal{I}}\right)$ la quasi-complétion de l'espace $\left(E_{0}, \mathscr{I}_{E_{0}}\right)$ (pour la Définition cf [18] p. 295-298).

Sur $F_{0}$ on définit également une topologie d'E.L.C. que l'on désignera $\operatorname{par} \mathscr{I}_{F_{0}}$.

Si $E_{1}$ et $F_{1}$ désignent des sous-espaces vectoriels de $E_{0}$ et $F_{0}$ respectivement, on désignera par $\sigma_{0}\left(E_{1}, F_{1}\right)$ (resp. $\sigma_{0}\left(F_{1}, E_{1}\right)$ ) la topologie induite sur $E_{1}$ (resp $F_{1}$ ) définie par la famille de semi-normes

$$
\left\{\left|\left(e_{1}, f_{1}\right)_{0}\right| \mid f_{1} \in F_{1}\right\} \quad\left(\operatorname{resp}\left\{\left|\left(e_{1}, f_{1}\right)_{0}\right| \mid e_{1} \in E_{1}\right\}\right) .
$$

De façon similaire si $E_{2}$ et $F_{2}$ désignent deux sous-espaces vectoriels de $E$ et $F$ respectivement on désignera par $\sigma\left(E_{2}, F_{2}\right)$ et $\sigma\left(F_{2}, E_{2}\right)$ les topologies localement convexes définies à l'aide des familles de semi-normes $\left\{\left|\left(e_{2}, f_{2}\right)\right| \mid f_{2} \in F_{2}\right\}$ et $\left\{\left|\left(e_{2}, f_{2}\right)\right| \mid e_{2} \in E_{2}\right\}$, sur $E_{2}$ et $F_{2}$ respectivement.

On supposera que:

$$
(u, v)=(u, \widetilde{v})_{0} \quad \forall[u, v] \in E_{0} \times F .
$$

Si $(A, \mathscr{I})$ désigne un E.V.T. $(A, \mathscr{I})^{\prime}$ désignera son dual (autrement l'ensemble des fonctionnelles linéaires continues sur $A$, pour $\mathscr{I}$ ).

On dira d'une application d'un E.V.T. dans un autre qu'elle est compacte, si elle transforme les bornés du premier dans les relativement compacts du second.

Si $X$ désigne un sous ensemble de $E_{0}$, on désignera par $\widehat{X}$ son quasicomplété dans $\widehat{E}_{0}$.

\section{2. Hypothèses de Base}

On supposera toujours dans la suite que 
1. $\widehat{E}_{0} \subseteq E$

2. $\left(E_{0},\|\|_{E_{0}} \hookrightarrow(E, \sigma(E, F))\right.$

3. Il existe $p$ linéaire continu de $(F, \sigma(F, E)) \rightarrow\left(F_{0}, \sigma_{0}\left(F_{0}, E_{0}\right)\right)$.

Précisons déjà qu'en général $p$ sera pas supposé injectif.

Il convient d'essayer de voir, dès maintenant, à quoi correspondent ces hypothèses dans la pratique. En effet, dans le pratique $E, E_{0}, F, F_{0}$ seront toujours des espaces normés, tels que $E$ et $F$ soient en dualité ainsi que $E_{0}$ et $F_{0}$.

Dans la pratique, il existera toujours une injection

$$
j:\left(E_{0}, \sigma_{0}\left(E_{0}, F_{0}\right)\right) \rightarrow(E, \sigma(E, F))
$$

et à ce moment-là, $p$ jouera le rôle $p=j^{*}:(F, \sigma(F, E)) \rightarrow\left(F_{0}, \sigma_{0}\left(F_{0}, E_{0}\right)\right)$. En général $p$ sera rarement injectif, et jouera souvent le rôle d'une projection. Pour cette raison, dans toute la suite on se servira, par analogie avec [14] de la notation:

$$
p(v)=\widetilde{v} \text { pour tout } v \in F
$$

Remarque 1.1. Signalons de plus, que lorsque $p$ sera une injection, il nous sera toujours possible d'identifier $F$ à un sous-ensemble de $F_{0}$, et à ce moment-là, à la place de $p(v)$, on écrira tout simplement $v$.

D'autre part l'hypothèse de base 1 ) nous permet d'identifier $\widehat{E}_{0}$ et donc $E_{0}$ à des sous ensembles de $E$, ce que nous ferons toujours dans la suite.

\section{3. Exemples de Situations}

Premiere Categorie. On suppose que $E=E_{0}, F=F_{0}$ et que $E$ contient un ensemble convexe $\sigma(E, F)$ compact absorbant. Il est facile de voir qu'alors, en choisissant $\mathscr{I}_{E_{0}}=\sigma(E, F)$, on a identité entre $E, E_{0}$, et $\widehat{E}_{0}$, et que les hypothèses de base sont realisées, lorsque l'on choisit pour $p$ l'identité.

De plus il est clair que cette situation englobe toutes les situations envisagées par H. Brezis [1] et en particulier tous les cas réflexifs.

Seconde Categorie. Soit $\left(Y, Y_{0} ; Z, Z_{0}\right)$ un système complémentaire au sens de [6] et [12] et $E_{1}$ et $F_{1}$ deux espaces normés en dualité notée $(,)_{1}$ (avec $Z$ et $F_{1}$ inclus dans un même e.v.t. $V$.) On choisit 


$$
\begin{array}{ll}
E_{0}=Y_{0} \cap E_{1} & F_{0}=Z+F_{1} \\
E=Y & F=Z_{0} .
\end{array}
$$

On suppose que $E_{0}$ et $F_{0}$ sont mis en dualité par une dualité $(,)_{0}$ s'identifiant a $(,)_{1}$ sur le couple $\left(Y_{0} \cap E_{1}\right) \times F_{1}$ et s'identifiant à la dualité entre $Y$ et $Z$ sur le couple $\left(Y_{0} \cap E_{1}\right) \times Z$.

On choisit alors

$$
\begin{array}{ll}
\|\|_{E_{0}}=\|\|_{Y / E_{0}} & \mathscr{I}_{F_{0}}=\sigma_{0}\left(Z+F_{1}, Y_{0} \cap E_{1}\right)=\sigma_{0}\left(F_{0}, E_{0}\right) \\
& \mathscr{I}_{E_{0}}=\sigma\left(Y, Z_{0}\right) .
\end{array}
$$

Il est clair de la définition d'un système complémentaire que les hypothèses de base $1,2,3$ sont realisées.

Remarquons que cette seconde catégorie englobe tous un ensemble de cas particuliers. En effet: choisissons $E_{1}=Y_{0}$ et $F_{1}=Z$ et on retrouve alors toutes les situations envisagées dans Donaldson [6] et Gossez [12]

Troisieme Categorie. Soient $B$ et $B_{0}$, deux espaces normés. On choisit

$$
E_{0}=B_{0}^{\prime} \quad E=B^{\prime} \quad F=B \quad F_{0}=B_{0}
$$

en supposant que

$$
B_{0}^{\prime} \hookrightarrow B^{\prime} \hookrightarrow B \hookrightarrow B_{0}
$$

et que $\quad \begin{cases}(v, u)_{0}=(v, u)_{B_{0}{ }^{\prime} \times B_{0}} & \text { pour tout }[u, v] \in B_{0} \times B_{0}^{\prime} \\ (v, u)=(v, u)_{B^{\prime} \times B} & \text { pour tout }[u, v] \in B \times B^{\prime}\end{cases}$

et on suppose que

$$
(v, u)_{0}=(v, u) \quad \text { pour tout }[u, v] \in E_{0} \times F .
$$

On choisit ensuite

$$
\|\|_{E_{0}}=\|\|_{B^{\prime} / B_{0}^{\prime}} .
$$

Il est clair alors que les hypothèses de base sont satisfaites.

Signalons que cette dernière catégorie englobe les situations envisagées dans les applications de Hess [14] et en fait plus, puisque dans [14] les espaces sont tous supposés séparables et réflexifs ce que nous n'exigeons pas ici. En particulier, cette situation englobera les cas envisagés par Browder [3] et Bui An Ton [23]. 
On peut évidemment envisager tout ensemble d'autres situations possibles. Nous ne le ferons pas ici puisque ces trois catégories suffisent à donner une idée des cas envisageables.

Dans les applications nous verrons des situations plus pratiques de ces trois situations.

\section{4. Définition des Opérateurs}

Avant de passer à la définition proprement dite des opérateurs, donnons quelques notations supplémentaires dont nous nous servirons par la suite.

Soient $\left\{\left[u_{\alpha}, v_{\alpha}\right]\right\}_{\alpha \in A}$ un net de $E_{0} \times F_{0}$ et $H$ un sous espace vectoriel de $E_{0}$. On notera brièvement par (I) les eventuelles propriétés de ce net

$$
\left\{\begin{array}{l}
\text { a) }\left\|u_{\alpha}\right\|_{E_{0}} \leq C \text { pour tout } \alpha \in A \text { et } u_{\alpha} \rightarrow u \text { dans }\left(\widehat{E}_{0}, \widehat{\mathcal{I}}\right) \\
\text { b) }\left\{\begin{array}{l}
v_{\alpha} \rightarrow \widetilde{v} \text { suivant } \mathscr{I}_{F_{0}} \text { et suivant } \sigma_{0}\left(F_{0}, H\right) \\
\text { pour un certain } v \in F
\end{array}\right. \\
\text { c) } \lim \sup \left(v_{\alpha}, u_{\alpha}\right)_{0} \leq(v, u) .
\end{array}\right.
$$

On désigne par $(1-H),(2-H),(3-H)$ et $(4-H)$ les propriétés suivantes d'un opérateur $T$ à domaine $D(T) \subseteq E$ et à valeurs dans $2^{F_{0}}$.

$$
\left\{\begin{array}{c}
\text { Pour tout } x \in H \subseteq D(T), T(x) \text { est un convexe compact de } \\
\left(F_{0}, \sigma_{0}\left(F_{0}, E_{0}\right)\right) .
\end{array}\right.
$$

$$
\begin{gathered}
\text { (2-H) } \quad\left\{\begin{array}{l}
\text { Pour tout } E_{i} \text {, sous-espace vectoriel de demension finie de } \\
H \subseteq D(T) \text { la restriction de } T \text { à } E_{i} \text { (que l'on notera } \\
\text { toujours } \left.T / E_{0}\right) \text { est demicontinue supérieurement de } E_{i} \\
\text { dans }\left(F_{0}, \sigma_{0}\left(F_{0}, E_{0}\right)\right) .
\end{array}\right. \\
(3-\mathrm{H}) \quad\left\{\begin{array}{l}
\mathrm{Si}\left\{\left[u_{\alpha}, v_{\alpha}\right]\right\}_{\alpha \in A} \text { est un net de } G r T \cap\left(H \times F_{0}\right) \text { vérifiant } \\
\text { les conditions (I) alors }[u, \widetilde{\gamma}] \in G r T .
\end{array}\right. \\
(4-\mathrm{H}) \quad\left\{\begin{array}{l}
\mathrm{Si}\left\{\left[u_{\alpha}, v_{\alpha}\right]\right\}_{\alpha \in A} \text { est un net de } G r T \cap\left(H \times F_{0}\right) \text { vérifiant } \\
\text { les conditions (I) alors }[u, \widetilde{\sigma}] \in G r T \text { et }\left(v_{\alpha}, u_{\alpha}\right)_{0} \rightarrow(v, u) .
\end{array}\right.
\end{gathered}
$$

On peut maintenant introduire la définition suivante:

Définition 1. 4. 1. Soient $\left(F_{0}, \mathscr{I}_{F_{0}}, F, E_{0},\|\|_{E_{0}}, \mathscr{I}_{E_{0}}, E\right)$ donnés et $H_{1}$ 
un sous espace vectoriel de $E_{0}$. Si $T$ est un opérateur de $D(T) \subseteq E \rightarrow 2^{F_{0}}$ on dit que $T$ est de type

$-(M)_{0}$ sur $\left(H_{1}\right)$ s'il satisfait aux conditions $(1-H),(2-H)$, et $(3-H)$ avec $H=H_{1}$.

$-(G P)_{0}$ sur $\left(H_{1}\right)$ s'il satisfait aux conditions $(1-H),(2-H)$ et $(4-H)$ avec $H=H_{1}$.

Les énoncés séquentiels de $(3-H)$ et $(4-H)$ seront notés $(3-H)$ s et $(4-H)_{s}$ respectivement et les opérateurs correspondants seront dit de type $(M)_{o s}$ et $(G P)_{o s}$ respectivement.

Notation. Dans toute la suite, si $T$ est un opérateur à domaine dans $E$ et à valeurs dans $F$, on lui associera un opérateur à valeurs dans $F_{0}$, noté $\widetilde{T}$ de la façon suivante:

$$
D(\widetilde{T})=D(T) \text { et } \widetilde{T}(x)=p(T(x)) .
$$

Nous allons maintenant donner quelques résultats permettant d'affirmer que sous certaines conditions l'ensemble des opérateurs de type (GP)。 ou $(G P)_{o s}$ reste stable par addition. Mais auparavant énonçons certaines propriétés éventuelles d'un opérateur $T: D(T) \subseteq E \rightarrow 2^{F}$.

$$
\begin{aligned}
& \left\{\begin{array}{l}
\text { La restriction } T / E_{0} \text { de } T \text { à } E_{0} \text { est compacte de }\left(E_{0},\|\|_{E_{0}}\right) \\
\text { dans }(F, \sigma(F, E)) .
\end{array}\right. \\
& \left\{\begin{array}{l}
\text { Si } X_{1} \text { est borné dans }\left(E_{0},\|\|_{E_{0}}\right) \text { et } C \text { est une constante } \\
\text { l'ensemble: } \\
\left\{v \in F \mid v \in T u \text { pour } u \in X_{1} \text {, et }(\widetilde{\gamma}, u)_{0} \leq C\right\} \\
\text { est relativement compact dans }(F, \sigma(F, E)) .
\end{array}\right.
\end{aligned}
$$

Et la propriété suivante d'un opérateur $T: E \rightarrow 2^{F_{0}}$

$$
\left\{\begin{array}{l}
\text { Si } X_{1} \text { est borné dans }\left(E_{0},\|\|_{E_{0}}\right) \text { alors l'ensemble } \\
\qquad\left\{(v, u)_{0} \mid u \in X_{1}, v \in T u\right\} \\
\text { est borné inférieurement. }
\end{array}\right.
$$

On a alors:

Proposition 1. 4. 2. Supposons l'application p injective et $\sigma_{0}\left(F_{0}, E_{0}\right)$ $\supseteq \mathscr{I}_{F_{0}}$, soient $R: E \rightarrow 2^{F}$ et $S: E \rightarrow 2^{F_{0}}$ deux opérateurs tels que: 


$$
\begin{aligned}
& -D(R) \cap D(S) \supseteq E_{0} \\
& -\widetilde{R} \text { et } S \text { vérifient }\left(4-E_{0}\right) \\
& -R \text { satisfait à (1). }
\end{aligned}
$$

Alors l'opérateur $T=\widetilde{R}+S: E \rightarrow 2^{F_{0}}$ vérifie également $\left(4-E_{0}\right)$.

Démonstration: Soit $\left\{\left[u_{\alpha}, v_{\alpha}\right]\right\}_{\alpha \in A}$ un net de $G r T \cap\left(E_{0} \times F_{0}\right)$ vérifiant (I).

On peut tout d'abord écrire

$$
v_{\alpha}^{T}=\widetilde{\gamma}_{\alpha}^{R}+v_{\alpha}^{S} \quad \text { avec } \quad v_{\alpha}^{R} \in R\left(u_{\alpha}\right) \quad \text { et } \quad v^{S} \in S\left(u_{\alpha}\right) .
$$

Puisque $\left\{u_{\alpha}\right\}$ est borné dans $\left(E_{0},\|\|_{E_{0}}\right)$ et que $R$ possède la propriété (1), alors $\left\{v_{\alpha}^{R}\right\}$ est relativement compact dans $(F, \sigma(F, E))$. Ainsi on peut extraire de $\left\{v_{\alpha}^{R}\right\}$ un sous-net $\left\{v_{\beta}^{R}\right\}$ convergeant vers $v^{R} \in F$ suivant $\sigma(F, E)$ et donc tel que $\left\{\widetilde{\boldsymbol{v}}_{\beta}^{R}\right\}$ converge vers $\widetilde{\boldsymbol{v}}^{R}$ suivant $\sigma_{0}\left(F_{0}, E_{0}\right)$.

Mais il découle alors que

$$
v_{\beta}^{S}=v_{\beta}^{T}-\widetilde{v}_{\beta}^{R} \rightarrow v^{S}=v^{T}-\widetilde{v}^{R} \in F
$$

suivant $\sigma_{0}\left(F_{0}, E_{0}\right)$ et donc suivant $\mathscr{I}_{F_{0}}$.

Nous allons maintenant envisager deux éventualités

-la première: supposons que

$$
\lim \sup \left(\widetilde{\gamma}_{\beta}^{R}, u_{\beta}^{R}\right)_{0} \leq\left(v^{R}, u\right)
$$

D'après la propriété $\left(4-E_{0}\right)$ de $\widetilde{R}$, on en déduit que $\widetilde{\boldsymbol{v}}^{R} \in \widetilde{R}(u)$ et que

$$
\left(\widetilde{\gamma}_{\beta}^{R}, u_{\beta}^{R}\right)_{0} \rightarrow\left(v^{R}, u\right)
$$

Mais ceci entraine que

$$
\begin{aligned}
\left(v^{S}, u\right) & =\left(v^{T}, u\right)-\left(v^{B}, u\right) \geq \lim \sup \left(v_{\beta}^{T}, u_{\beta}\right)_{0}-\lim \left(\widetilde{v}_{R}^{R}, u_{\beta}^{R}\right) \\
& =\lim \sup \left(v_{\beta}^{S}, u_{\beta}\right) .
\end{aligned}
$$

A nouveau grâce à la propriété $\left(4-E_{0}\right)$ de $S$, on conclue que $v^{s} \in S(u)$ et que $\left(v_{\beta}^{S}, u_{\beta}\right) \rightarrow\left(v^{s}, u\right)$, ainsi

$$
v^{T} \in T(u) \text { et }\left(v_{\beta}^{T}, u_{\beta}\right) \rightarrow\left(v^{T}, u\right) .
$$

-la seconde: supposons que $\lim \sup \left(v_{\beta}^{R}, u_{\beta}\right)_{0}>\left(v^{R}, u\right)$. Intervertissant les rôles de $\widetilde{R}$ et de $S$ dans la partie précédente, on obtient que

$$
\lim \sup \left(v_{\beta}^{s}, u_{\beta}\right)_{0}>\left(v^{s}, u\right)
$$


on peut alors extraire un sous-net tel que

$$
\left(\widetilde{\boldsymbol{v}}_{r}^{R}, u_{\tau}\right)_{0} \rightarrow\left(v^{R}, u\right)+a \text { avec } a>0 .
$$

Mais pour la même raison que précédemment, on aura encore

$$
\lim \sup \left(\widetilde{\boldsymbol{v}}_{r}^{\boldsymbol{R}}, u_{\tau}\right)_{0}>\left(\boldsymbol{v}^{S}, u\right)
$$

par conséquent, quitte à extraire une nouvelle un sous-net, on peut affirmer que

$$
\begin{cases}\left(\widetilde{\gamma}_{r}^{R}, u_{r}\right)_{0} \rightarrow\left(v^{R}, u\right)+a & a>0 \\ \left(v_{r}^{S}, u_{r}\right)_{0} \rightarrow\left(v^{S}, u\right)+b & b>0 .\end{cases}
$$

Donc

$$
\left(v_{r}^{T}, u_{r}\right)_{0} \quad\left(v^{T}, u\right)+a+b
$$

contredisant l'hypothèse

$$
\lim \sup \left(v_{\alpha}^{T}, u_{\alpha}\right)_{0} \leq\left(v^{T}, u\right) .
$$

Notre second résultat sur les sommes d'opérateurs est contenu dans la proposition suivante.

Proposition 1. 4. 3. Supposons l'application p injective et que $\mathfrak{I}_{F_{0}}$ $C \sigma_{0}\left(F_{0}, E_{0}\right)$ et soient $R: E \rightarrow 2^{F}$ et $S: E \rightarrow 2^{F_{0}}$ deux opérateurs tels que:

$$
\begin{aligned}
& -\widetilde{R}+S \text { satisfont à }\left(4-E_{0}\right) \\
& -D(R) \cap D(S) \supseteq E_{0} \\
& -R \text { vérifie la condition (2) } \\
& -S \text { vérifie la condition (3). }
\end{aligned}
$$

Alors l'opérateur $T=\widetilde{R}+S: E \rightarrow 2^{F_{0}}$ satisfait également à $\left(4-E_{0}\right)$.

Nous ne donnerons pas ici la démonstration de cette proposition très voisine de celle de la proposition précédente. Une démonstration dans un cas particulier peut être trouvée dans [27].

Nous avons jusqu'à maintenant donné deux résultats concernant des opérateurs mettant en jeu' des nets. Nous allons à présent énoncer deux résultats parallèles concernant des opérateurs ayant trait à des suites. Mais pour cela nous aurons besoin avant tout de quelques définitions. 
Définition 1. 4. 4. Soit $A$ un ensemble muni d'une topologie $I$. On dira que l'espace topologique $(A, \mathscr{I})$ possède la propriété $\left(s_{1}\right)$ si tout sous-ensemble compact de $(A, \mathscr{I})$ est également séquentiellement compact.

En ce qui concerne certains exemples de tels espaces, intéressants dans le cadre des opérateurs non-linéaires, on pourra se referrer à l'Apendice I de [27].

On désigne, en parallèle avec (1) et (2), par (1) s et (2) s les propriétés éventuelles d'un opérateur $T: E \rightarrow 2^{F}$

(1) $)_{s}\left\{\begin{array}{l}\mathrm{Si}\left\|u_{n}\right\|_{E_{n}} \leq C,\left\{u_{n}\right\} \subseteq D(T) \text { et }\left\{u_{n}\right\} \text { converge dans }\left(\widehat{E}_{0}, \widehat{I}\right) \text { alors } \\ T\left(\left\{u_{n}\right\}\right) \text { est relativement compact dans }(F, \sigma(F, E)) .\end{array}\right.$ (2). $\left\{\begin{array}{l}\text { Si }\left\{u_{n}\right) \subseteq D(T),\left\|u_{n}\right\|_{E_{0}} \leq C \text { et }\left\{u_{n}\right\} \text { converge dans }\left(\widehat{E}_{0}, \widehat{\mathcal{I}}\right) \text { et } \\ C_{1} \text { est une constante, l'ensemble } \\ \quad\left\{v \in F \mid v \in T u_{n} \text {, pour un certain } n \text {, et }\left(\widetilde{v}, u_{n}\right)_{0} \leq C_{1}\right\} \\ \text { est relativement compact dans }(F, \sigma(F, E)) .\end{array}\right.$

Et par (3), l'analogue de (3) concernant $T: D(T) \subseteq E \rightarrow 2^{F_{0}}$

(3). $\left\{\begin{array}{c}\mathrm{Si}\left\{u_{n}\right\} \subseteq D(T),\left\|u_{n}\right\|_{E_{0}} \leq C \text { et }\left\{u_{n}\right\} \text { converge dans }\left(E_{0}, \mathcal{I}\right) \\ \text { l'ensemble }\left\{\left(v, u_{n}\right)_{0} \mid v \in T u_{n}\right\} \text { est borné inférieurement. }\end{array}\right.$

On peut maintenant énoncer deux résultats parallèles aux Propositions 1.4.2 et 1.4 .3 respectivement.

Proposition 1. 4. 5. Supposons $p$ injective et $\mathscr{I}_{F_{0}} \subseteq \sigma_{0}\left(F_{0}, E_{0}\right)$. Supposons également que $\left(F_{0}, \mathscr{I}_{F_{0}}\right)$ possède la propriété $\left(s_{1}\right)$ et soient $R: E \rightarrow 2^{F}$ et $S: E \rightarrow 2^{F_{0}}$ deux opérateurs tels que

$$
\begin{aligned}
& -D(R) \cap D(S) \supseteq E_{0} \\
& -\widetilde{R} \text { et } S \text { satisfont } \grave{a}\left(4-E_{0}\right)_{s} \\
& -R \text { satisfait } \grave{a}(1)_{s} .
\end{aligned}
$$

Alors l'opérateur $T=\widetilde{R}+S: E \rightarrow 2^{F_{0}}$ vérifie également $\left(4-E_{0}\right)_{\mathrm{s}}$.

Et de même

Proposition 1. 4. 6. Supposons $p$ injective, $\mathscr{I}_{F_{0}} \subseteq \sigma_{0}\left(F_{0}, E_{0}\right)$ et que 
$\left(F_{0}, \mathcal{I}_{F_{0}}\right)$ possède la proprieté $\left(s_{1}\right)$. Soient $R: E \rightarrow 2^{F}$ et $S: E \rightarrow 2^{F_{0}}$ deux opérateurs tels que:

$$
\begin{aligned}
& -D(R) \cap D(S) \supseteq E_{0} \\
& -\widetilde{R} \text { et } S \text { satisfont à }\left(4-E_{0}\right)_{s} \\
& -R \text { remplit la condition }(2)_{s} \\
& -S \text { remplit la condition }(3)_{s} .
\end{aligned}
$$

Alors l'opérateur $T=\widetilde{R}+S$ vérifie également $\left(4-E_{0}\right)_{8}$.

Les démonstrations de ces deux résultats sont analogues à celles des Propositions 1.4 .2 et 1.4 .3 , aux modifications près dûes à l'utilisation des suites à la place de nets. Nous les omettrons donc.

Il convient également de noter que de tous ces résultats, desquels découlent immédiatement des résultats sur les sommes d'opérateurs de type $(G P)$ ou $(G P)_{0}$, des conditions particulières ont été exigées tant du côté des espaces que du côté des opérateurs. On pourrait aisément donner d'autres résultats parallèles tout en modifiant ces conditions par tout un ensemble de variantes.

Soulignons, qu'en particulier, les Propositions 1.4.2, 1.4.3, 1.4.5 et 1.4.6, généralisent tous les résultats correspondants de [27] et donc de Browder-Hess [4] et certains résultats de Gossez [12].

\section{Chapitre 2. Résultats de Surjectivité pour les Opérateurs de Type $(M)_{0}$ où $(M)_{0 s}$}

Signalons dès à présent que des résultats de surjectivité plus complets ayant trait aux opérateurs plus particuliers de type $(G P)_{0}$, avec des conditions de coercivité affaiblies font l'objet du chapitre suivant.

Ce chapitre se subdivise en deux paragraphes. Le premier est reservé à des résultats généraux avec des conditions de coercivité du type de celles de Brezis [1]. Le second à la traduction de certains de ces résultats dans le cadre des situations des seconde et troisième catégorie du chapitre précédent.

Soulignons également que tous les résultats d'existence que nous donnerons par la suite s'écrivent sans difficulté supplémentaire dans le cas 
de topologies plus générales, de ce fait nous donnerons assez souvent des énoncés se prettant aisément à cette transcription.

\section{1. Coercivite Ordinaire}

Avant tout nous aurons besoin d'une notation, empruntée à Brezis [1]. Si $X$ et $Y$ désignent deux ensembles convexes d'un espace localement convexe $E$, on désigne par $\operatorname{Int}_{Y} X$ l'ensemble

$$
\text { Int }_{y} X=\left\{x \in X \mid Y-x \subseteq \bigcup_{\lambda \geq 0} \lambda(Y-x)\right\} \text {. }
$$

Il est clair que $\operatorname{Int}_{X} X=X$ et que $\operatorname{Int}_{E} X$ représente très exactement l'intérieur de $X$ pour la topologie localement convexe la plus fine sur $E$.

On énonce le premier résultat d'existence

Théorème 2. 1. 1: Supposons l'injection $\left(E_{0},\|\|_{E_{0}}\right) \hookrightarrow\left(\widehat{E}_{0}, \widehat{\mathscr{I}}\right)$ compacte et $\mathscr{I}_{F_{0}} \subseteq \sigma_{0}\left(F_{0}, E_{0}\right)$. Soit $X$ un ensemble convexe fermé borné absorbant de $\left(E_{0},\|\|_{E_{0}}\right)$ et $T$ un opérateur de $D(T) \subseteq E \rightarrow 2^{F_{0}}$ tel que:

1. $D(T) \supseteq E_{0}$

2. T est de type $(M)_{0}$ sur $\left(E_{0}\right)$

3. T remplisse la condition:

(C1) $\quad(v, u)_{0} \geq 0$ pour tout $[u, v] \in G r T \cap\left(E_{0} \times F_{0}\right)$ tel que $u \notin \operatorname{Int}_{E_{0}} X$.

Alors l'ensemble $S=\{u \in \widehat{X} \mid 0 \in T u\}$ est non vide.

Démonstration: Soit $\Lambda_{0}$ le filtre des sous-espaces de dimension finie $E_{\alpha}$ de $E_{0}$. Designons par $j_{\alpha}$ l'injection

$$
j_{\alpha}: E_{\alpha} \rightarrow\left(E_{0}, \sigma_{0}\left(F_{0}, E_{0}\right)\right)
$$

et $j_{\alpha}^{*}$ son adjointe par rapport au couple en dualité $\left(E_{0}, F_{0}\right)$

$$
j_{\alpha}^{*}:\left(F_{0}, \sigma_{0}\left(F_{0}, E_{0}\right)\right) \rightarrow E_{\alpha}^{*} .
$$

Désignons de plus, par $T_{\alpha}$ l'opérateur de $E_{\alpha} \rightarrow 2^{E_{\alpha}^{\prime}}$ défini par

$$
T_{\alpha}=j_{\alpha}^{*} T j_{\alpha} .
$$

On vérifie aisément que cet opérateur satisfait aux hypothèses du Lemme 2.2 de [12], et par conséquent, pour tout $\alpha$, il existe $u_{\alpha} \in X \cap E_{\alpha}$ et $v_{\alpha} \in T u_{\alpha}$ tel que 


$$
j_{\alpha}^{*} v_{\alpha}=0 .
$$

Mais cette dernière équation entraine, en particulier, que $v_{\alpha} \rightarrow 0$ suivant $\sigma_{0}\left(F_{0}, E_{0}\right)$ et par conséquent suivant $\mathcal{I}_{F_{0}}$.

D'autre part, $X$ est relativement compact dans $\left(\widehat{E}_{0}, \widehat{I}\right)$ et par conséquent on peut extraire de $\left\{u_{\alpha}\right\}$ un sous-net $\left\{u_{\beta}\right\}$ tel que $\left[u_{\beta}, v_{\beta}\right] \rightarrow$ $[u, 0] \in \widehat{E}_{0} \times F_{0}$ suivant $\widehat{\mathcal{I}} \times \mathscr{I}_{F_{0}}$. Comme de par ailleurs

$$
\left(v_{\beta}, u_{\beta}\right)_{0}=0
$$

il en découle immédiatement par le type $(M)_{0}$ de $T$ que $[u, 0] \in G r T$.

C.Q.F.D.

Dans le théorème précédent nous avons supposé que $\mathcal{I}_{F_{0}} \subseteq \sigma_{0}\left(F_{0}, E_{0}\right)$. Que peut-on dire dans le cas où cette hypothèse ne serait pas remplie? Une réponse partielle à cette question est contenue dans le résultat suivant:

Théorème 2. 1. 2. Supposons l'injection $\left(E_{0},\|\|_{E_{0}}\right) \hookrightarrow\left(\widehat{E}_{0}, \widehat{\mathcal{I}}\right) \mathrm{com}$ pacte et.que $\sigma_{0}\left(F_{0}, E_{0}\right) \subseteq \mathcal{I}_{F_{0}}$. Soit $X$ un ensemble convexe fermé borné absorbant dans $\left(E_{0},\|\|_{E_{0}}\right)$.

Soit $T$ un opérateur de $D(T) \subseteq E \rightarrow 2^{F_{0}}$ tel que

1. $D(T) \supseteq E_{0}$

2. T est de type $(M)_{0}$ sur $\left(E_{0}\right)$

3. T satisfasse à la condition (C1)

4. T satisfasse à la condition suivante.

$$
\left\{\begin{array}{l}
\text { Si } X_{1} \text { désigne un ensemble borné de }\left(E_{0},\|\|_{E_{0}}\right) \text { et } C \\
\text { une constante, l'ensemble } \\
\quad\left\{v \in F_{0} \mid v \in T u \text {, pour un certain } u \in X_{1} \text {, et }(v, u)_{0} \leq D\right\} \\
\text { est relativement compact dans }\left(F_{0}, I_{F_{0}}\right) .
\end{array}\right.
$$

Alors l'ensemble $S=\{u \in \widehat{x} \mid 0 \in T u\}$ est non vide.

Démonstration: On démontre comme dans le. théorème précédent l'existence d'un net $\left[u_{\alpha}, v_{\alpha}\right] \in G r T$ tel que $u_{\alpha} \in X \cap E_{\alpha}$ et

$$
j_{\alpha}^{*} v_{\alpha}=0
$$


Ainsi $v_{\alpha} \rightarrow 0$ suivant $\sigma_{0}\left(F_{0}, E_{0}\right)$. Mais $T$ satisfait à la condition (4), et ainsi par la relative compacité de $X$ dans $\left(\widehat{E}_{0}, \widehat{I}\right)$ on peut extraire de $\left[u_{\alpha}, v_{\alpha}\right]$ un sous-net $\left[u_{\alpha}, v_{\beta}\right]$ tel que

$$
\left[u_{\beta}, v_{\beta}\right] \rightarrow[u, v] \in \widehat{E}_{0} \times F_{0} \text { suivant } \widehat{\mathcal{I}} \times \mathscr{I}_{F_{0}} .
$$

Mais $\sigma_{0}\left(F_{0}, E_{0}\right) \subseteq \mathscr{I}_{F_{0}}$ et par conséquent $v=0$, et on termine la démonstration comme dans le théorème précédent.

C.Q.F.D.

Les deux résultats précédents concernent des opérateurs du type $(M)_{0}$. En ce qui concerne les opérateurs de type $(M)_{08}$ on peut donner deux résultats parallèles. Mais avant de les énoncer nous aurons besoin de la:

Définition 2. 1. 3. Soit $E_{1}$ un espace localement convexe. On dit que $E_{1}$ possède la propriété $(s)$ si de tout net $\left\{x_{\alpha}\right\}$ borné dans $E_{1}$ et convergeant vers $x$, on peut en extraire $\left\{x_{n}\right\}$, suite ordinaire, convergeant également vers $x$.

Pour de plus amples détails concernant cette propriété et les espaces qui la possèdent nous renvoyons à [27].

Et notons par (5) la propriété éventuelle suivante d'un opérateur $T: E \rightarrow 2^{F_{0}}$.

$$
\left\{\begin{array}{l}
\text { Si } X_{1} \text { désigne un sous ensemble borné de }\left(E_{0}, \mathscr{I}_{1}, E_{0}\right) \\
\text { et } C \text { une constante, l'ensemble } \\
\quad\left\{v \in F_{0} \mid v \in T u \text {, pour } u \in X_{1} \text {, avec }(v, u)_{0} \leq C\right\} \\
\text { est relativement compact dans }\left(F_{0}, \sigma_{0}\left(F_{0}, E_{0}\right)\right) .
\end{array}\right.
$$

On est maintenant en mesure de formuler les deux énoncés séquentiels des Théorèmes 2.1 .1 et 2.1.2, respectivement.

Théorème 2. 1. 4. Supposons l'injection $\left(E_{0},\|\|_{E_{0}}\right) \hookrightarrow\left(\widehat{E}_{0}, \widehat{\mathcal{I}}\right)$ compacte et que $\mathcal{I}_{F_{0}} \subseteq \sigma_{0}\left(F_{0}, E_{0}\right)$. Supposons de plus que l'espace $\left(\widehat{E}_{0}, \widehat{I}\right)$ possède la propriété $\left(s_{1}\right)$ et que l'espace $\left(F_{0}, \mathscr{I}_{F_{0}}\right)$ possède la propriété (s).

Soit $X$ un ensemble convexe fermé borné et absorbant de 
$\left(E_{0},\|\|_{E_{0}}\right)$ et $T: D(T) \subseteq E \rightarrow 2^{F_{0}}$ un opérateur vérifiant

1. $D(T) \supseteq E_{0}$

2. T est de type $(M)_{0 s}$ sur $\left(E_{0}\right)$

3. T satisfait aux conditions (C1) et (4).

Alors l'ensemble $S=\{u \in \mathbb{X} \mid 0 \in T u\}$ est non vide.

Et de façon similaire

Théorème 2. 1. 5. Supposons l'injection $\left(E_{0},\|\|_{E_{0}}\right) \hookrightarrow(\widehat{E}, \widehat{\mathscr{I}}) \mathrm{com}$ pacte et $\sigma_{0}\left(F_{0}, E_{0}\right) \subseteq \mathcal{I}_{F_{0}}$. Supposons de plus que l'espace $\left(\widehat{E}_{0}, \widehat{\mathcal{I}}\right)$ possède la propriété $\left(s_{1}\right)$ et que $\left(F_{0}, \sigma_{0}\left(F_{0}, E_{0}\right)\right)$ possède la propriété $(s)$.

Soit $X$ un ensemble convexe fermé borné et absorbant de ( $E_{0}$, \|\|$\left._{E_{0}}\right)$ et $T: D(T) \subseteq E \rightarrow 2^{F_{0}}$ un opérateur vérifiant

1. $D(T) \supseteq E_{0}$.

2. $T$ est de type $(M)_{0 s}$ sur $\left(E_{0}\right)$.

3. $T$ satisfait aux conditions (C1) et (5).

Alors l'ensemble $S=\{u \in \widehat{X} \mid 0 \in T u\}$ est non vide.

Remarque. Il est clair, de l'énoncé des quatre théorèmes précédents, qu'on peut jouer sur les hypothèses afin d'obtenir tout un ensemble de variantes.

Notons de plus, que dans les quatre résultats précédents nous avons supposé que $E_{0} \subseteq D(T)$. Que peut-on dire dans le cas où cette hypothèse ne serait pas remplie. Nous allons donner ici un résultat dans le cas ou $D(T) \supseteq H$ sous-espace vectoriel de $E_{0}$, "assez grand".

Nous laissons le soin au lecteur d'écrire sa formulation séquentielle et d'énoncer tout un ensemble de résultats voisins. Le cas très important où le domaine de $T$ sera un ensemble bien plus général, fera l'objet d'un travail ultérieur. On a ainsi le:

Théorème 2. 1.6. Soit $\mathscr{I}_{0}$ une nouvelle topologie localement convexe définie sur $E_{0}$. On note $\left(E_{0}{ }^{*}, \mathscr{I}_{0}{ }^{*}\right)$ le dual fort de $\left(E_{0}, \mathscr{I}_{0}\right)$ et on suppose que $F_{0} \subseteq E_{0}{ }^{*}$. Supposons de plus l'injection $\left(E_{0},\|\|_{E_{0}}\right)$ $\hookrightarrow\left(\widehat{E}_{0}, \widehat{I}\right)$ compacte, $\mathcal{I}_{F_{0}} \subseteq \sigma_{0}\left(F_{0}, E_{0}\right)$ et que 


$$
(v, u)_{0}=(v, u)_{E_{0} * \times E_{0}} \text { pour tout }[u, v] \in E_{0} \times F_{0} \text {. }
$$

Soient $X$ un ensemble convexe fermé borné et absorbant de ( $E_{0}$, \|\|$\left._{E_{0}}\right)$ et $T: D(T) \subseteq E \rightarrow 2^{F_{0}}$ un opérateur tel que:

1) $D(T) \cap E_{0} \supseteq H$, sous-espace vectoriel dense dans $\left(E_{0}, \mathcal{I}_{0}\right)$

2) $T$ est de type $(M)_{0}$ sur $(H)$

3) $T$ satisfait à la condition

(C2): $(v, u)_{0} \geq 0$ pour tout $[u, v] \in G r T \cap\left(H \times F_{0}\right)$ tel que $u \notin \operatorname{Int}_{H}(H \cap X)$

4) $T$ satisfait à la condition:

(6)

$$
\left\{\begin{array}{l}
\text { Si } X_{1} \text { est borné dans }\left(E_{0},\|\|_{E_{0}}\right) \text { l'ensemble } \\
\qquad\left\{v \in F_{0} \mid v \in T u \text { pour } u \in X_{1} \text {, avec }(v, u)_{0} \leq C\right\} \\
\text { est equicontinu dans }\left(E_{0}{ }^{*}, \mathcal{I}_{0}{ }^{*}\right)
\end{array}\right.
$$

Alors l'ensemble $S=\{u \in \widehat{X} \mid 0 \in T u\}$ est non vide.

Nous ne donnons pas ici la démonstration de ce résultat, puisqu'elle s'apparente fortement à celle des résultats précédemment énoncés ici, aux modifications près dûes à ces hypothèses moins restrictives sur le domaine de l'opérateur.

Rajoutons au passage que ces théorèmes ainsi que leurs conséquences généralisent les résultats d'existence connus sur les opérateurs de type (M) dont le domaine contient un sous-espace vectoriel "assez grand". En particulier, ils généralisent les résultats parallèles de Brezis [1], Browder-Hess [4], Hess [14], Gossez [12], Donaldson [5].

\section{2. Théorèmes de Surjectivité Globale}

Nous appliquons ici les résultats du chapitre précédent aux deux dernières catégories de situation envisagées dans le premier chapitre. Les principaux résultats concernant la première catégorie ont déjà été donnés dans [27].

Seconde Categorie. On choisit les espaces et les topologies comme dans le chapitre I, comme conséquence du Théorème 2.1.1 on a entre autres 
Proposition 2. 2. 1. Soit $T: E \rightarrow 2^{F_{0}}$ vérifiant

1) $D(T) \supseteq E_{0}$

2) $T$ est de type $(M)_{0}$ sur $\left(E_{0}\right)$

3) $T$ satisfait à la condition de coercivité: $\forall f \in Z_{0}$

(C3) $\left.\lim _{\|u\|_{Y_{0} \rightarrow+\infty}}\left\{\inf \left|(v-\tilde{f}, u)_{0}\right|[u, v] \in G r T \cap\left(Y_{0} \cap E_{1}\right) \times\left(Z+F_{1}\right)\right]\right\}=+\infty$. Alors $R(T) \supseteq Z_{0}$.

Et du Théorème 2.1.6 on déduit la

Proposition 2. 2. 2. On choisit pour $\mathcal{I}_{0}$ la topologie de la norme $\|u\|_{Y_{0} \cap E_{1}}=\|u\|_{Y_{0}}+\|u\|_{F_{1}}$ et on suppose que le dual $E_{0}{ }^{*}$ de $E_{0}$ pour cette norme vérifie $F_{0} \subseteq E_{0}{ }^{*}$ et

$$
(v, u)_{0}=(v, u)_{E_{0}^{*} \times E_{0}} \quad \forall[u, v] \in E_{0} \times E_{0} .
$$

Soit $T: E \rightarrow 2^{F}$ 。érifiant:

1) $D(T) \supseteq H$ sous-espace vectoriel de $E_{0}$ dense dans $\left(E_{0},\|\|_{Y_{0} \cap E_{1}}\right)$

2) $T$ est de type $(M)_{0}$ sur $(H)$

3) $T$ satisfait à (C3) et à (6) pour $\mathscr{I}_{0}$ définie comme ci-dessus. Alors $R(T) \supseteq Z_{0}$.

Troisieme Categorie. On choisit ici aussi les espaces et les topologies comme dans le premier chapitre. Du Théorème 2.1.1 il découle alors:

Proposition 2. 2. 3. Soit $T: E \rightarrow 2^{F_{0}}$ vérifiant

1) $D(T) \supseteq E_{0}$

2) $T$ est de type $(M)_{0}$ sur $\left(E_{0}\right)$

3) $T$ satisfait à la condition de coercivité: $\forall f \in B$

$$
\lim _{\|\|_{B^{\prime}}}\left\{\inf \left|(v-\tilde{f}, u)_{0}\right| \mid[u, v] \in G r T \cap\left(B_{0}{ }^{\prime} \times B_{0}\right)\right\}=+\infty .
$$

Alors $R(T) \supseteq B$.

Et du Théorème 2.1.6 entre autres la:

Proposition 2. 2. 4. Soit $\mathscr{I}_{0}=\sigma\left(B_{0}{ }^{\prime}, B_{0}\right)$ et $H$ un sous-espace 
vectoriel $\sigma\left(B_{0}{ }^{\prime}, B_{0}\right)$ dense dans $B_{0}{ }^{\prime}$.

Soit $T: B_{0}^{\prime} \rightarrow 2^{B_{0}}$ tel que

1) $H \subseteq D(T)$

2) $T$ est de type $(M)_{0} \operatorname{sur}(H)$

3) $T$ satisfait à (C4) et à (6) pour $\mathscr{I}_{0}=\sigma\left(B_{0}{ }^{\prime}, B_{0}\right)$.

Alors $R(T) \supseteq B$.

Remarque. On notera que les conditions (C3) et (C4) sont des conditions de coercivité affaiblies, essentielles dans certaines applications (cf Application 4.1 où la condition de coercivité forte habituelle n'est pas réalisée).

\section{Chapitre 3. Methodes d' Homotopies}

Dans le chapitre précédent nous avons établi certains résultats d'existence concernant des opérateurs non linéaires. Cependant, ces résultats, quoique concernant également les opérateurs de type $(G P)_{0}$, restent spécifiques des opérateurs de type $(M)_{0}$ par leurs structures et leurs conditions. Par ailleurs, au cours des dernières années plusieurs auteurs, et parmi eux, Browder, Hess, Wille, Gossez,..., se sont aperçus que dans le cas particulier des opérateurs de type "pseudomonotone" on pouvait affaiblir nettement certaines conditions dans les théorèmes d'existence et surtout la condition de coercivité, et ce, en utilisant des méthodes d"'homotopie”.

Nous nous proposons, dans ce chapitre, de montrer que ces méthodes s'étendent aisément au cadre présent, et par là même, renforcent les résultats de Browder [3], Hess [14] [15] [17], Gossez [12] [13], Fitzpatrick [8], Wille [26],...

\section{1. Homotopies Pseudomonotones et Théorèmes d'Existence}

Avant tout signalons quelques notations utiles par la suite. Soit $A$ un sous-ensemble d'un E.L.C. On note $\bar{A}$ la fermeture de $A$, et $\partial A$ sa frontière. Si $X$ est un sous-ensemble de $E_{0}$, on notera $\partial_{0} X$ (resp. $\stackrel{\circ}{X}$ ) sa frontière (resp. son intérieur) dans $\left(E_{0},\|\|_{E_{0}}\right)$.

On supposera dans tout ce chapitre l'injection de $\left(E_{0},\|\|_{E_{0}}\right) \subset\left(\widehat{E}_{0}, \widehat{I}\right)$ 
compacte et que $F_{0}$ est muni d'une norme notée \|\|$_{F_{0}}$.

Commençons par énoncer un résultat important dans la suite

Lemme 3. 1. 1. Soit $E_{1}$ un espace vectoriel de dimension finie, $A$ un voisinage de 0 dans $E_{1}$, convexe symétrique et borné. Soit $T$ : $\bar{A} \times[0,1] \rightarrow 2^{\mathbb{R}^{*}}$ tel que:

1) T est demicontinu supérieurement

2) Pour tout $(x, t) \in \bar{A} \times[0,1], T(x, t)$ est convexe

3) Pour tout $x \in \partial A$, et $\lambda>0 T(x, 1) \cap \lambda T(-x, 1)=\phi$

4) $0 \notin T(\partial A, t)$ pour tout $t \in[0,1]$.

Alors pour tout $t \in[0,1]$, on a $0 \in T(A, t)$

Démonstration: Il suffit évidemment de montrer que $0 \in T(A, 0)$. Comme on peut identifier $E_{1}{ }^{*}$ à $E$, les conditions du lemme assurent que $T(x, 0)$ et $T(x, 1)$ sont homotopes dans $C(\partial A, \bar{A}, 0)$ au sens de [25]. Le résultat découle alors de Théorèmes (10.1) et (9.3) de ce dernier article.

Corollaire 3.1.2. Soient $E_{1}$ et $A$ comme dans le lemme ci-dessus et soit $T$ satisfaisant à 1), 2) et 4) du lemme précédent et de plus à l'une des deux conditions suivantes

- Soit $T(x, 1)$ est impaire sur $\partial A($ i.e. $-T(x, 1)=T(-x, 1))$

- Soit $(v, x)>0$ pour tout $[x, v] \in G r T_{1}$ avec $x \in \partial A$.

Démonstration: Ce Corollaire set une conséquence immédiate du lemme précédent puisque chacune des deux conditions entraine en particulier 3) du Lemme 3.1.1.

On introduit maintenant une liste de propriétés éventuelles d'un opérateur $T: D(T) \subseteq E \times[0,1] \rightarrow 2^{F_{0}}$. $(5-\mathrm{H}) \quad\left\{\begin{array}{l}\text { Pour tout }[x, t] \in H \times[0,1], T(x, t) \text { est un convexe compact } \\ \operatorname{de}\left(F_{0}, \sigma_{0}\left(F_{0}, H\right)\right) .\end{array}\right.$

(6-H) $\left\{\begin{array}{l}\text { Pour tout } E_{i} \text { sous-espace de dimension finie de } H, T /_{E_{i} \times[0,1]} \\ \text { est demicontinu lorsque } F_{0} \text { est muni de la topologie } \sigma_{0}\left(F_{1}, H\right) .\end{array}\right.$ 
Soit $\left\{\left[u_{\alpha}, t_{\alpha}, v_{\alpha}\right]\right\}$ un net de $H \times[0,1] \times F_{0}$. On note (II) les propriétés

$$
\left\{\begin{array}{l}
\text { (a) }\left\|u_{\alpha}\right\|_{E_{0}} \leq C \text { et } u_{\alpha} \rightarrow u \text { dans }\left(\widehat{E}_{0}, \widehat{\mathcal{I}}\right) \\
\text { (b) } v_{\alpha} \rightarrow \widetilde{v} \text { dans }\left(F_{0}, \mathcal{I}_{r_{0}}\right) \text { pour un certain } v \in F \\
\text { (c) } t_{\alpha} \rightarrow t \\
\text { (d) } \lim \sup \left(v_{\alpha}, u_{\alpha}\right)_{0} \leq(v, u) .
\end{array}\right.
$$

Et:

$(7-\mathrm{H}) \quad\left\{\begin{array}{c}\text { Pour tout net }\left\{\left[u_{\alpha}, t_{\alpha}, v_{\alpha}\right]\right\} \text { de } G r T \cap\left(H \times[0,1] \times F_{0}\right) \\ \text { satisfaisant à (II) on } a:[u, t] \in D(T), v \in T(u, t) \text { et } \\ \left(v_{\alpha}, u_{\alpha}\right)_{0} \rightarrow(v, u) .\end{array}\right.$

Toutes les propriétés analogues énoncées au moyen de suites, seront notées de façon identique, avec une indexation par la lettre " $s$ ".

On est en mesure alors d'introduire la:

Définition 3. 1. 3. Soit $T: D(T) \supseteq E \times[0,1] \rightarrow 2^{F_{0}}$. On dit que $T$ constitue une homotopie pseudomonotone (resp. s-pseudomonotone) sur $\left(H_{1}\right)$ s'il satisfait à:

1) $D(T) \supseteq H_{1} \times[0,1]$

2) $T$ satisfait à $(5-\mathrm{H})$ et $(6-\mathrm{H})$ avec $H=H_{1}$

3) $T$ satisfait à $(7-\mathrm{H})\left(\operatorname{resp}(7-\mathrm{H})_{s}\right)$ avec $H=H_{1}$

On aura également besoin de la

Définition 3. 1. 4. Soit $T$ une homotopie pseudomonotone sur $(H)$. On dit que $T$ possède la propriété $(N+)$ sur un sous ensemble $X$ de $E_{0}$ si pour tout net $\left\{\left[u_{\alpha}, t_{\alpha}, v_{\alpha}\right]\right\} \subseteq G r T$ tel que:

1) $\left\{u_{\alpha}\right\} \subseteq \partial_{0} X$ et $u_{\alpha} \rightarrow u$ dans $\left(\widehat{E}_{0}, \widehat{\mathcal{I}}\right)$

2) $t_{\alpha} \rightarrow t \neq 0$

3) $v_{\alpha} \rightarrow \widetilde{v}$ dans $\left(F_{0}, \mathscr{I}_{F_{0}}\right)$ pour $v \in F$

4) $\left(v_{\alpha}, u_{\alpha}\right)_{0} \rightarrow(v, u)$.

Alors $0 \notin T(u, t)$.

On note $(S+)$ la propriété analogue énoncée au moyen de suites. 
Remarque: Notons que la définition précédente est la généralisation naturelle au cadre présent de la propriété $(S+)$ introduite par Browder. Elle permet de plus de se dispenser d'utiliser des opérateurs de dualité, ou des opérateurs de dualité généralisés (cf Gossez [12]) qui n'existent pas toujours dans les situations que l'on peut avoir à considerer.

Le premier résultat d'existence dans les convexes est contenu dans le:

Théorème 3. 1. 5. Soit $X$ un convexe absorbant de $E_{0}$, fermé et borné dans $\left(E_{0},\|\|_{E_{0}}\right)$ et soit $T$ une homotopie pseudomonotone sur $\left(E_{0}\right)$ satisfaisant $\grave{a}$ :

1) $T$ vérifie $(N+)$ sur $X$

2) $T(X \times[0,1])$ est relativement compact dans $\left(F_{0}, \mathcal{I}_{F_{0}}\right)$

3) $0 \notin T\left(\partial_{0} X, t\right)$ pour tout $t \in[0,1]$

4) $T$ satisfait à l'une des deux conditions suivantes:

$-T(-x, 1)=-T(x, 1)$ pour $x \in \partial_{0} X$

$-(v, x)_{0}>0$ pour $[x, v] \in G r T_{1}$ avec $x \in \partial_{0} X$.

Alors $S_{t}=\{u \in \widehat{X} \mid 0 \in T(u, t)\}$ est non vide pour tout $t \in[0,1]$.

Démonstration: Il suffit évidemment de montrer que $S_{0} \neq \phi$. On s'inspire de Hess [14]. Soit $\Lambda_{0}$ le filtre des sous espaces $E_{i}$ de dimension finie de $E_{0}$ et $j_{i}$ l'injection de $E_{i} \rightarrow E_{0}$, de duale $j_{i}^{*}$ par rapport au couple $\left(E_{0}, F_{0}\right)$. Pour tout $i$ désignons par $X_{i}$ l'ensemble $X \cap E_{i}$ et par $T_{i}$ l'opérateur de $E_{i} \times[0,1] \rightarrow 2^{E_{i}^{*}}$ défini par

$$
T_{i}(x, t)=j_{i}^{*} T\left(j_{i} x, t\right) .
$$

Distinguons deux cas:

$1^{\text {er }}$ cas: il existe $E_{i_{0}} \in \Lambda_{0}$ tel que pour tout $E_{i} \supseteq E_{i_{0}}$, pour tout $u_{i} \in \partial_{0} X_{i}$ $=\partial_{0} X \cap E_{i}$ et pour tout $t \in[0,1], 0 \notin T_{i}\left(u_{i}, t\right)$. Alors grâce au Corollaire 3.1.2 il existe un net $\left\{u_{j}\right\}$ tel que $u_{j} \in X_{j}, 0 \in T_{j}\left(u_{j}, 0\right)$ pour tout $E_{j} \supseteq E_{i_{0}}$. La compacité relative de $X$ dans $\left(\widehat{E}_{0}, \widehat{\mathcal{I}}\right)$ et la pseudomonotonie de $T$ entrainent que tout point limite $u$ de $\left\{u_{j}\right\}$ pour $\widehat{\mathcal{I}}$ vérifie $0 \in T(u, 0)$. 
$2^{e}$ cas: il existe un sous-net de sous-espaces de dimension finie $\left\{E_{\alpha}\right\}$ de $E_{0}$ et $\left\{\left[u_{\alpha}, t_{\alpha}\right]\right\} \subseteq H \times[0,1]$ tels que:

$$
u_{\alpha} \in \partial_{0} X_{\alpha} \text { et } 0 \in T_{\alpha}\left(u_{\alpha}, t_{\alpha}\right) \text { et } t_{\alpha} \rightarrow t_{0} \text {. }
$$

Comme on peut supposer que $u_{\alpha} \rightarrow u$ suivant $\widehat{\mathcal{I}}, t_{\alpha} \rightarrow t_{0}$ et trouver $v_{\alpha} \in T\left(u_{\alpha}, t_{\alpha}\right)$ avec

$$
j_{\alpha}^{*} v_{\alpha}=0 \text { et } v_{\alpha} \rightarrow v_{0} \text { dans }\left(F_{0}, \mathscr{I}_{F_{0}}\right)
$$

en conséquence, $\left(v_{\alpha}, u_{\alpha}\right)_{0}=0$ et $v_{\alpha} \rightarrow 0$ dans $\left(F_{0}, \sigma_{0}\left(F_{0}, E_{0}\right)\right)$, donc $v_{0}=0$. De la pseudomonotonie de $T$, il s'ensuit alors que $0 \in T\left(u, t_{0}\right)$.

On affirme maintenant que $t_{0}=0$. En effet s'il en était autrement, par la propriété $(N+)$ de $T$ on en déduirait une contradiction. Donc $0 \in T(u, 0)$.

C.Q.F.D.

Remarques: 1) Dans le théorème précédent on aurait pu affaiblir les hypothèses sur l'homotopie de $T$, et entre autres, ne supposer que $T$ soit pseudomonotone que sur $X \cap H$ où $H$ est un sous-espace vectoriel de $E_{0}$, moyennant des hypothèses analogues à celles du Théorème 2.1.6.

2) On peut énoncer dans tout ce chapitre des résultats similaires pour les s-pseudomonotones.

3) On peut énoncer des théorèmes analogues aux propositions du premier chapitre, permettant d'affirmer que la somme de deux homotopies pseudomonotones est une homotopie pseudomonotone.

Par ailleurs, il est intéressant de noter que dans le cas où les opérateurs $T_{t}$ sont positifs, on peut affaiblir les hypothèses du théorème précédent grâce au

Theoreme 3. 1.6. Soit $X$ un convexe fermé absorbant de ( $E_{0}$, \|\|$\left._{E_{0}}\right)$ et soit $T: D(T) \subseteq E \times[0,1] \rightarrow 2^{F_{0}}$ tel que:

1) Pour tout $t \in] 0,1]$ fixé, $T_{t}: E \rightarrow 2^{F_{0}}$ soit de type $(M)_{0}$ sur $\left(E_{0}\right)$

2) Pour tout $t \in] 0,1], T_{t}$ satisfasse $\grave{a}$ (C1): $(v, u)_{0} \geq 0$ pour tout $[u, v] \in G r T_{t} \cap\left(E_{0} \times F_{0}\right)$ tel que $u \notin \operatorname{Int}_{B_{0}} X$ et (4): L'ensemble $\left\{v \in F_{0} \mid v \in T_{t} u\right.$ pour un certain $u \in X$, avec (v, $u_{0}$ $\leqq C\}$ est relativement compact dans $\left(F_{0}, \mathscr{I}_{F_{0}}\right)$ 
3) L'ensemble $\underset{\left.t \in \mathcal{J}_{0}, 1\right]}{\bigcup}\left\{T_{t}^{-1}(0)\right\}$ est relativement compact dans $\left(\widehat{E}_{0}, \widehat{\mathscr{I}}\right)$

4) Pour tout $f \in F$ et tout net $\left\{\left[u_{\alpha}, t_{\alpha}\right]\right\}$ tel que $u_{\alpha} \rightarrow u$ dans $\left(\widehat{E}_{0}, \widehat{\mathcal{I}}\right), t_{\alpha} \rightarrow 0$ et $f \in T\left(u_{\alpha}, t_{\alpha}\right)$, on $a: u \in D\left(T_{0}\right)$ et $f \in T_{0}(u)$. Alors $S=\left\{u \in \widehat{X} \mid 0 \in T_{0}(u)\right\} \neq \phi$.

Démonstration: Il découle immédiatement du Théorème 2.1.2 que pour tout $0<t \leq 1$, il existe $u_{t} \in D\left(T_{t}\right)$ avec $0 \in T_{t}\left(u_{t}\right)$. Faisant tendre $t$ vers 0 , grâce à la condition 3 ) on peut supposer que $u_{t} \rightarrow u_{0}$ dans $\left(\widehat{E}_{0}, \widehat{I}\right)$. Le résultat découle alors trivialement de 4$)$.

Remarquons que l'on s'est dispensé dans le résultat précédent des hypothèses classiques des homotopies.

\section{2. Solutions Globales et Conditions Affaiblies}

Dans le paragraphe précédent nous avons dans le Théorème d'existence 3.1.5 supposé d'une part que $0 \notin T\left(\partial_{0} X, t\right), \forall t \in[0,1]$ et que $X$ était donné. Ces deux points peuvent ne pas être connus au préalable. Utilisant une idée de Gossez [12], nous allons ici donner un résultat permettant de surmonter ces difficultés.

Commençons tout d'abord par une variante du Lemme 3.1.1.

Lemme 3. 2. 1. Soit $E_{1}$ un espace vectoriel de dimension finie et $T: E_{i} \times[0,1] \rightarrow 2^{E_{1}^{*}}$ un opérateur tel que

1) $T(x, t)$ est convexe pour tout $(x, t) \in E_{i} \times[0,1]$

2) $T$ est demicontinu supérieurement

3) Pour tout $y \in E_{i}^{*}$ il existe $V^{\prime}(y)$ un voisinage de $y$ tel que $\bigcup_{t \in[0,1]} T_{t}^{-1}\left(V^{\prime}\right)$ soit borné dans $E_{i}$

4) $T_{1}$ vérifie l'une des deux hypothèses suivantes:

-soit $T_{1}$ est impaire a l'exterieur d'une certaine boule $B_{E_{t}}(0, R)$

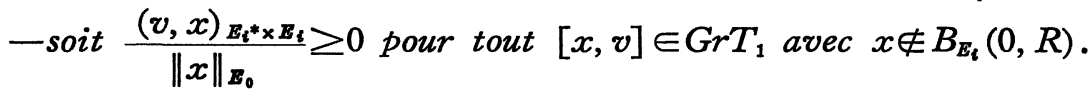
Alors $R\left(T_{t}\right)=E_{i}^{*}$ pour tout $t \in[0,1]$.

Démonstration: Signalons avant tout que ce lemme ne découle pas 
immédiatement du Théorème 3.8 de [12] contrairement aux apparences. En effet, la démonstration de ce dernier résultat est etablie dans le cas spécifique d'espaces de dimension infinie.

De plus il est clair qu'il suffit montrer que $0 \in R\left(T_{0}\right)$. Or grâce à la condition 3) il existe $R>0$ tel que $0 \notin T_{t}(x)$ pour tout $(x, t) \in S_{E_{i}}$ $(0, R) \times[0,1]$. Comme on peut identifier $E_{1}{ }^{*}$ à $E_{1}$, il découle de [25] que $T_{0}$ et $T_{1}$ sont homotopes dans $C\left(S_{E_{i}}(0, R), B_{E_{i}}(0, R), 0\right)$. On peut supposer $R$ assez grand pour que chacune des conditions 4 ) entraine que $T_{1}(x) \cap \lambda T_{1}(-x)=\phi$, pour $\lambda>0$ et $x \in S_{E_{i}}(0, R)$. Le résultat découle alors des Théorèmes (9.3) et (10.1) de [25] par exemple.

Signalons que le lemme précédent englobe la Proposition 10 de Browder-Hess [4].

Mais sa conséquence importante est contenue dans le

Theoreme 3.2.2. Soit $T$ une homotope pseudomonotone sur $\left(E_{0}\right)$ possdant la proprieté $(N+)$ sur les boules de $E_{0}$ et satisfaisant à

1) $T$ satisfait à (C5):

$$
\left\{\begin{array}{l}
\text { Pour tout } f \in F \text { il existe un voisinage } V_{0}(\tilde{f}) \text { dans } \\
\left(F_{0},\|\|_{F_{0}}\right) \text { tel que } \\
\bigcup_{t \in[0,1]} T_{t}^{-1}\left(V_{0}\right)
\end{array}\right.
$$

est borné dans $\left(E_{0},\|\|_{E_{0}}\right)$.

2) $T_{1}$ vérifie l'une des deux conditions:

- soit $T_{1}$ est impaire à l'exterieur d'une boule de $\left(E_{0},\|\|_{E_{0}}\right)$

- soit $\inf \left\{\frac{(v, x)_{0}}{\|x\|_{E_{0}}} \mid v \in T_{1}(x)\right\}_{\|x\|_{\mathbb{E}_{0}} \rightarrow+\infty} \rightarrow+\infty$.

Alors $R\left(T_{t}\right) \supseteq p(F)$ pour tout $t \in[0,1]$.

Démonstration: Elle est construite sur une combinaison naturelle des idées utilisées dans la demonstration du lemme 3.2.1 et du Théorème 3.1. 5 .

Soulignons que le théorème précédent contient en particulier tout les résultats similaires de Browder [3], Hess [14], [17], Gossez [12] 
[13], Fitzpatrick [8],...

Il convient de préciser qu'à priori le Théorème 3.2.2 ne recouvre pas, dans le cas où l'homotopie $T_{t}$ remplit la condition de coercivité de 2), tous les cas de Gossez [12]. Mais en fait grâce au corollaire suivant, la généralisation est complète.

Corollaire 3. 2.3. Soient $T$ et $J$ deux homotopies pseudomonotones sur $E_{0}$ avec $J$ possedant la proprieté $(N+)$ sur les boules de $E_{0}$ et telles que pour tout $\varepsilon>0 H_{\varepsilon}=T+\varepsilon J$ soit également une homotopie pseudomonotone sur $\left(E_{0}\right)$. On suppose

1) Pour tout $\varepsilon>0, H_{\varepsilon}$ satisfait à (C5)

2) Pour tout $\varepsilon>0 H_{\varepsilon}(., 1)$ satisfait à

$$
\inf \left\{\frac{(v, x)_{0}}{\|x\|_{E_{0}}} \mid v \in H(x, 1)\right\}_{\|x\|_{\mathbb{E}_{0}} \rightarrow+\infty} \rightarrow+\infty
$$

3) Pour tout $\tilde{f} \in p(F)$ l'ensemble $\underset{\alpha<c_{<}<\varepsilon_{0}}{S} H_{\varepsilon}^{-1}(\tilde{f})$ est relativement compact dans $\left(\widehat{E}_{0}, \widehat{\mathcal{I}}\right)$ et il existe $C>0$ tel que

$$
\left|(v, u)_{0}\right| \leq C \quad \forall[u, v] \in S \times J(S \times[0,1]) .
$$

Alors $R\left(T_{t}\right) \supseteq p(F)$ pour tout $t \in[0,1]$.

Démonstration: C'est une conséquence immédiate du théorème précédent. Puisque $\forall \varepsilon>0, R\left(H_{\varepsilon}\right) \supseteq p(F)$, il suffit de faire tendre $\varepsilon \rightarrow 0$. Le résultat découle alors de 4 ).

\section{3. Applications Théoriques}

Il est intéressant d'énoncer dans les situations envisagées au premier chapitre ce que deviennent les résultats précédents.

Par exemple pour la seconde catégorie le Corollaire 3.2.3 donne un résultat important dans la pratique de la manière suivante:

Soit $\left(Y_{1}, Y_{0}, Z_{1}, Z_{0}\right)$ un système complémentaire et $E_{1}$ et $F_{1}$ comme définis dans le premier chapitre, paragraphe 1.3 et soit $T_{t}$ une homotopie pseudomonotone au sens de Gossez [12], et $J$ une application de dualité de $Y \rightarrow 2^{Z}$ au sens de [12]. On associe à $\varepsilon J+T_{t}$ la famille 
d'opérateurs $\varepsilon \widetilde{J}+\widetilde{T}_{t}$ définie par

$$
\varepsilon \tilde{J}+\widetilde{T}_{t}=\left(\varepsilon \widetilde{J+T_{t}}\right)=p o\left(\varepsilon J+T_{t}\right)
$$

on suppose $E_{1}$ dense dans $Y_{0}$, pour la norme de $Y_{0}$ et que $T_{t}$ satisfasse à la condition (cf [12])

$$
\left\{\begin{array}{l}
\text { chaque } z_{0} \in Z_{0} \text { possède un voisinage } V\left(z_{0}\right) \text { tel que } \\
\bigcup_{t \in[0,1]} T_{t}^{-1}(V) \text { soit borné dans } Y .
\end{array}\right.
$$

Alors on a:

Théorème 3. 3.1. Soit $P_{t}$ une homotopie pseudomonotone sur $\left(E_{1}\right)$ telle que

$$
\begin{gathered}
-\left(P_{t}(x), x\right)_{0} \geq 0 \quad \forall t \in[0,1] \text { et } \forall x \in E_{0} . \\
-\left\{y \in F_{1} \mid y \in P_{t}(x) \quad \text { pour }(x, t) \in E_{1} \times[0,1],\|x\|_{E_{0}} \leq C\right. \text { et } \\
\left.(y, x) \leq C\|x\|_{E_{0}}\right\}
\end{gathered}
$$

est relativement compact dans $\sigma\left(F_{0}, E_{0}\right)$.

Alors $H_{\varepsilon}=\tilde{J}+\widetilde{T}_{t}+P_{t}$ est une homotopie pseudomonotone sur $\left(E_{1}\right)$ satisfaisant aux conditions du Corollaire 3.2.3. En particulier si $T_{1}$ vérifie

$$
(v, x) \geq 0 \quad \forall[x, v] \in G r T \text { avec }\|x\|_{E_{0}}>R>0
$$

on a $R\left(\widetilde{T}_{t}+P_{t}\right) \supseteq p(F)$.

Démonstration: Que $H_{\varepsilon}$ satisfasse à (C5) découle immédiatement de la positivité de $T_{1}$ et $P_{t}$ et d'un argument similaire à celui utilisé dans l'application 4.1 (indépendant).

La condition 2) du Corollaire 3.2.3 est immédiate. Quant à la condition 3) elle s'obtient comme (C5) tenant compte du fait que $T_{t}$ possède la propriété (C6).

C.Q.F.D.

Une autre application importante pour des situations de la troisième catégorie. Soient donc $B$ et $B_{0}$ comme dans le paragraphe 1.3 avec les mêmes topologies. On suppose juste de plus que $B=X^{\prime}$ où $X$ est un 
espace de Banach tel que $B_{0}^{\prime} \hookrightarrow X$.

On rappelle (cf [29]) qu'un graphe monotone $\bar{M}$ de $X^{\prime \prime} \times X^{\prime}$ est dit $J$-approximable s'il existe une famille d'opérateurs de type $(G P)_{0}$ sur $(X)$ (pour les topologies $\sigma\left(X^{\prime \prime}, X^{\prime}\right)$ et $\sigma\left(X^{\prime}, X\right)$ ) notée $\left\{\bar{M}_{\lambda}\right\}$ tels que si $J_{x}$ désigne l'application de dualité de $X \rightarrow 2^{X^{\prime}}$ alors pour tout $x \in X$, et tout $\lambda>0$ il existe $\left[x_{\lambda}^{* *}, x_{\lambda}^{*}\right] \in G r \bar{M}$ tel que $x_{\lambda}^{*} \in \bar{M}_{\lambda} x$ et

$$
0 \in J_{x}\left(x_{\lambda}^{* *}-x\right)+\lambda x_{\lambda}^{*} \text {. }
$$

De plus si $\left\|x_{\lambda_{a}}^{* *}\right\|_{X^{*}} \leq C$ et $\lambda_{\alpha} \rightarrow 0$ avec:

$\left\{\begin{array}{l}{\left[x_{\lambda}^{* *}, x_{\lambda_{\alpha}}^{*}\right] \in G r \bar{M}_{\lambda_{\alpha}} \text { et }\left[x_{\lambda}^{* *}, x_{\lambda_{\alpha}}^{*}\right] \rightarrow\left[x_{\lambda}^{* *}, x_{0}^{*}\right] \text { suivant } \sigma\left(X^{\prime \prime}, X^{\prime}\right) \times \sigma\left(X^{\prime}, X\right)} \\ \text { et } \lim \sup \left(x_{\lambda_{\alpha}}^{*}, x_{\alpha}^{* *}\right)_{X^{\prime} \times X^{*}} \leq\left(x_{0}^{*}, x_{0}^{* *}\right)_{X^{\prime} \times X^{\prime \prime}}\end{array}\right.$ on a $\left[x_{0}^{* *}, x_{0}^{*}\right] \in G r \bar{M}$ et $\left(x_{\lambda_{\alpha}}^{*}, x_{\alpha}^{* *}\right)_{X^{\prime} \times X^{*}} \rightarrow\left(x_{0}^{*}, x_{0}^{* *}\right)_{X^{*} \times X^{*}}$. On sait, entre autres, (cf [29]) que sous certaines conditions si $M$ est un opérateur monotone dans $X \times X^{\prime}$ possèdant une unique extension maximale monotone $\bar{M}$ à $X^{\prime \prime} \times X^{\prime}, \bar{M}$ est $\bar{J}$-approximable. Ainsi si $\psi$ est une fonction convexe propre sur, $X, \overline{\partial \psi}$ est $\bar{J}$-approximable (cf [29]).

On peut alors déduire des résultats précédents, la généralisation au cas non réflexif des Théorèmes 1 et 2 et leurs Corollaires de Hess [15] grâce au

Théorème 3. 3.2. Soit $T$ un opérateur de type $(G P)_{0}$ sur $\left(B_{0}{ }^{\prime}\right)$ satisfaisant à (3) et $\bar{M}$ un opérateur maximal monotone $\bar{J}$-approxim. able dans $X^{\prime \prime} \times X^{\prime}$ telle que $[0,0] \in \bar{M}$ et vérifiant

(7) $\left\{x^{*} \in X^{\prime} \mid x^{*} \in \bar{M}\left(x^{* *}\right)\right.$ avec $\left.\left(x^{*}, x^{* *}\right) \leq C\right\}$ est borné dans $X^{\prime}$

On associe à $\bar{M}$ l'opérateur $\widetilde{M}: E=X^{\prime \prime} \rightarrow 2^{F_{0}}$ par $\widetilde{M}=p o \bar{M}$. Alors si $T$ satisfait à

$$
\begin{aligned}
& \forall f \in F \\
& \lim _{\|u\|_{\mathbb{B}_{0} \rightarrow+\infty}}\left\{\inf (v-\tilde{f}, u)_{0} \mid[u, v] \in G r T \cap\left(E_{0} \times F_{0}\right)\right\}=+\infty
\end{aligned}
$$

on $a$ :

$$
R(T+\widetilde{M}) \supseteq F=X^{\prime}
$$

Démonstration: On associe aux opérateurs $\bar{M}_{\lambda}$, les opérateurs $\widetilde{M}_{\lambda}$ $=p o \bar{M}_{\lambda}$. Il est clair d'une variante de la Proposition 1.4.3 que $T+\widetilde{M}_{\lambda}$ est de type $(G P)_{0}$ sur $\left(E_{0}\right)$ et vérifie aussi $(C 7)$ puisque $[0,0] \in \bar{M}$. 
Ainsi pour tout $\lambda>0$, on a $R\left(T+\widetilde{M}_{2}\right) \supseteq F$. Ainsi grâce à la propriété (7) de $\bar{M}$, passant à la limite quand $\lambda \rightarrow 0$, on obtient le résultat.

Remarque. Comme on l'a signalé plus haut, ce résultat s'applique toujours à $\overline{\partial \psi}$. En particulier soit $K$ convexe fermé de $X$, d'intérieur contenant 0 et $\psi_{K}$ sa fonction indicatrice. On sait (cf [29]) que $\overline{\partial \psi}_{\mathbf{K}}$ possède la propriété (7). Appliquant le résultat précédent à $\overline{\partial \psi}_{K}=\bar{M}$ on obtient des solutions d'inéquations variationnelles recouvrant les résultats de Hess [15].

Il est important de signaler que le Corollaire 3.2.3 donne lieu à une extension du Théorème 2 de Hess [15] de la façon suivante. Soient $B_{1}, B_{0}$ et $X$ comme précédemment. On a:

Théorème 3. 3. 3. Soit $T: B^{\prime} \rightarrow 2^{B_{0}}$ un opérateur de type $(G P)_{0}$ sur $\left(B_{0}^{\prime}\right)$ tel que:

1) il existe $u_{0} B_{0}^{\prime}$ avec

$$
\inf _{\substack{[u, v] \in G r T \\ u \in B_{0}^{\prime}}}\left\{\frac{\left(v, u-u_{0}\right)}{\|u\|_{B^{\prime}}}+\|v\|_{B_{0}}\right\}_{\|u\|_{X_{0} \rightarrow+\infty} \rightarrow+\infty} \rightarrow+\infty
$$

2) l'ensemble

$$
\left\{v \in B_{0} \mid v \in T(u) \text { pour } u \in B_{0}{ }^{\prime}, \operatorname{avec}(v, u)_{0} \leq C\right\}
$$

est relativement $\sigma\left(B_{0}, B_{0}{ }^{\prime}\right)$ compact.

Alors $R(T) \supseteq B$.

On peut évidemment énoncer un résultat similaire généralisant le Théorème 3.5 et le Corollaire 3.7 de Gossez [12] au cadre du premier exemple théorique ci-dessus.

\section{Chapitre 4. Applications}

Les applications que nous donnons ici sont de deux types: d'une part des problèmes elliptiques et d'autre part des problèmes paraboliques. Les notations utilisées ici sont empruntées à Gossez [12] [13] et quelquefois Fougeres [9] [10]. Ainsi pour des détails supplémentaires nous renvoyons 
à ces travaux.

Soit $\Omega$ un ouvert de $\boldsymbol{R}^{n}$ et $M: \boldsymbol{R} \rightarrow \boldsymbol{R}$ vérifiant

$$
M(t)>0 \text { pour } t>0, \lim _{t \rightarrow+\infty} \frac{M(t)}{t}=+\infty, \lim _{t \rightarrow 0} \frac{M(t)}{t}=0
$$

on dit que $M$ est une $N$-fonction. On note $\bar{M}$ sa conjuguée.

On définit la classe d'Orlicz

$$
\begin{aligned}
& L_{M}(\Omega)=\{u \text { mesurable sur } \Omega \text { pour la mesure } \\
& \text { de Lebesque } \left.\mid \int_{\Omega} M(u(x)) d x<+\infty\right\} .
\end{aligned}
$$

Désignant par $L_{M}(\Omega)$ l'espace vectoriel engendré par $L_{M}(\Omega)$ on sait qu'il est de Banach pour la norme

$$
\|u\|_{(M)}=\inf \left\{k>0 \mid \int_{\Omega} M(u / k) d x \leq 1\right\} .
$$

On note $E_{M}(\Omega)$ la fermeture de $L^{\infty}(\Omega)$ dans $\left(L_{M}(\Omega)\right.$, \|\|$\left._{(M)}\right)$ et par $W^{m} L_{M}(\Omega)$ l'espace des fonctions ainsi que leurs derivées partielles d'ordre inférieur ou egale à $m$, à $L_{M}(\Omega)$. C'est un espace de Banach pour la norme

$$
\|u\|_{m, M}=\left(\sum_{|\alpha| \leq m}\left\|D^{\alpha} u\right\|_{(M)}^{2}\right)^{1 / 2}
$$

qui s'identifie à un sous-espace de $\pi_{|\alpha| \leq m} L_{M}(\Omega)$. On note $W^{m} E_{M}(\Omega)$ son sous-espace construit de façon analogue à partir de $E_{M}(\Omega)$.

On suppose dans toute la suite que $\Omega$ possède la propriété du segment (cf [12]) et on note $W_{0}^{m} L_{M}(\Omega)$ et $W_{0}^{m} E_{M}(\Omega)$ les fermetures de $D(\Omega)$ dans $W^{m} L_{M}(\Omega)$ pour les topologies $\|u\|_{m, M}$ et $\sigma\left(\pi L_{M}, \pi L_{\mathbb{H}}\right)$ respectivement.

On définit de façon similaire les espaces d'Orlicz-Sobolev anisotropes. (cf [9] et [12]). Soit $\alpha=\left(\alpha_{1}, \cdots, \alpha_{p}\right)$ un multi-indice et $|\alpha|=\alpha_{1}+\cdots$ $+\alpha_{p}$, et $\vec{M}=\left\{M_{\alpha}|| \alpha \mid \leq m\right\}$ une famille de $N$-fonctions, de famille duale $\vec{N}=\left\{\bar{M}_{\alpha}|| \alpha \mid \leq m\right\}$. On note

$$
W^{m} L_{\vec{M}}(\Omega)=\left\{u \mid D^{\alpha} u \in L_{M_{\alpha}}(\Omega), \text { pour tout }|\alpha| \leq m\right\}
$$

qui est un espace de Banach pour

$$
\|u\|_{m, \vec{\mu}}=\left(\sum_{|\alpha| \leq m}\left\|D^{\alpha} u\right\|_{\left(M_{\alpha}\right)}\right)^{1 / 2}
$$


On définit de façon analogue les espaces $W^{m} E_{\overrightarrow{\mathbb{M}}}(\Omega), W_{0}^{m} L_{\overrightarrow{\mathbb{R}}}(\Omega)$ et $W_{0}^{m} E_{\overrightarrow{\mathrm{M}}}(\Omega)$.

On pose également

$$
W^{-m} L_{M}(\Omega)=\left\{f \in \mathscr{D}^{\prime}(\Omega) \mid f=\sum_{|\alpha| \leq \mathbf{s} m}(-1)^{|\alpha|} D^{\alpha} f_{\alpha} \text { avec } f_{\alpha} \in L_{M}(\Omega)\right\}
$$

et

$$
W^{-m} E_{M}(\Omega)=\left\{f \in \mathscr{D}^{\prime}(\Omega) \mid f=\sum_{|\alpha| \leq m}(-1)^{|\alpha|} D^{\alpha} f_{\alpha} \text { avec } f_{a} \in E_{M}(\Omega)\right\} \text {. }
$$

Soit $M$ une $N$-fonction. On dit que $M$ satisfait à la condition $\Delta_{2}$ s'il existe $k>0$ tel que $M(2 t) \leq k M(t)$ pour $t$ assez grand.

\section{1. Première Application}

Nous nous proposons ici de résoudre dans un sens "convenable" des équations du type

$$
\widetilde{T} u+P u=f
$$

où $\widetilde{T}$ est un opérateur du type

$$
\widetilde{T} u=\sum_{|\alpha| \leq m}(-1)^{|\alpha|} D^{\alpha} T_{\alpha}\left(x, u, \cdots, \nabla^{m} u\right)
$$

et $P$ un opérateur du type

$$
P u=\sum_{|\alpha| \leq m-1}(-1)^{|\alpha|} D^{\alpha} P_{\alpha}\left(x, u, \cdots, \nabla^{m-1} u\right) .
$$

Les conditions imposées sur $\widetilde{T}$ sont empruntées en partie à Gossez [13], mais celle sur $P$ sont très différentes. Mais avant introduisons les notations suivantes:

$$
s_{m}=\operatorname{card}\left\{\alpha \in N^{n}|| \alpha \mid \leq m\right\} \quad s_{m}^{\prime}=\operatorname{card}\{\alpha \in N|| \alpha \mid=m\} .
$$

Si $\xi=\left\{\xi_{\alpha}|| \alpha \mid \leq m\right\} \in \boldsymbol{R}^{s_{m}}$ on note $\zeta=\left\{\xi_{\alpha}|| \alpha \mid=m\right\} \in \boldsymbol{R}^{s_{m^{\prime}}}$ et $\eta=\left\{\xi_{\alpha}|| \alpha \mid<m\right\}$ $\in R^{s_{m-1}}$ on note $\xi(u)=\left\{D^{\alpha} u|| \alpha \mid \leq m\right\}$.

Soit $M_{1}$ et $M_{2}$ deux $N$-fonctions telles que $M_{2}<M_{1}$ (cf [12]). On suppose que les coefficients $T_{\alpha}$ satisfont à (cf [12]).

(4. 1.3) Chaque $T_{\alpha}: \Omega \times \boldsymbol{R}^{s_{m}} \rightarrow \boldsymbol{R}$ est une fonction de $x$ mesurable pour $\xi$ fixé, et une fonction de $\xi$ continue pour $x$ fixé.

(4. 1. 4) Il existe des fonctions $a_{\alpha}(x) \in E_{M_{1}}(\Omega)$ pour $|\alpha|=m$ et à 
$L_{M_{1}}(\Omega)$ pour $|\alpha|<m$ et des constantes $C_{1}$ et $C_{2}$ positives telles que pour tout $(x, \xi) \in \Omega \times \boldsymbol{R}^{s_{m}}$

$$
\left|T_{\alpha}(x, \xi)\right| \leq a_{\alpha}(x)+C_{1 \beta \mid \zeta m} \sum_{\mid M^{-1} M\left(C_{2} \xi_{\beta}\right)}
$$

(4.1.5) Pour chaque $\left(x, \xi, \xi^{\prime}\right) \in \Omega \times \boldsymbol{R}^{s_{m}} \times \boldsymbol{R}^{s_{m}}$

$$
\sum_{|\alpha| \leq m}\left(T_{\alpha}(x, \xi)-T_{\alpha}\left(x, \xi^{\prime}\right)\right)\left(\xi_{\alpha}-\xi_{\alpha}^{\prime}\right) \geq 0
$$

Remarque. Ces conditions ne sont pas les plus faibles que l'on puisse imposer. D'autres conditions plus faibles sont envisagées dans l'application suivante et s'appliquent ici aussi.

(4. 1.6) Il existe une fonction $b(x) \in L^{1}(\Omega)$ et une constante positive $d$ telle que

$$
\sum_{|\alpha| \leq m} T_{\alpha}(x, \xi) \xi_{\alpha} \geq d \sum_{|\alpha| \leq m} M_{1}\left(\xi_{\alpha}\right)-b(x) .
$$

On choisit ensuite un espace normé $\left(E_{1},\|\|_{1}\right)$ tel que $D(\Omega) \subseteq E_{1}$ et que $\left(E_{1},\|\|_{1}\right) \hookrightarrow \mathscr{C}_{0}{ }^{m}(\Omega)=$ fonctions $m$ fois continument différentiables, nulles ainsi que leurs derivées sur $\partial \Omega\}$ et tel que $W^{-m} L_{\bar{M}_{1}}(\Omega) \hookrightarrow\left(E_{1}{ }^{*},\|\|_{1}{ }^{*}\right)$.

Remarque. Comme espace $E_{1}$, on peut choisir un espace du type $W_{0}^{p} L_{M_{s}}(\Omega)$ où $M_{z}$ est une $N$-fonction et $p$ un entier "assez grand" (pour des conditions précises ef [7]). En particulier, on peut choisir un espace de Sobolev ordinaire d'ordre assez grand. On peut aussi choisir $\mathscr{C}_{0}{ }^{m}(\Omega)$ lui-même.

De plus, on suppose que l'on a les injections continues suivantes:

$$
E_{1} \hookrightarrow W_{0}^{m} L_{M_{1}}(\Omega) \hookrightarrow W_{0}^{1} L_{M_{1}}(\Omega) \hookrightarrow L^{2}(\Omega) \hookrightarrow W^{-m} L_{\bar{M}_{1}}(\Omega) \hookrightarrow E_{1} *
$$

L'injection $W_{0}^{1} L_{M_{1}}(\Omega) \hookrightarrow L^{2}(\Omega)$ n'est pas du tout triviale lorsque $M_{1}$ ne satisfait pas à la condition $A_{2}$ ), cependant s'avère verifiée dans la grande partie des applications.

On choisit alors les espaces et les topologies suivantes:

$$
E_{0}=E_{1}, \quad E=W_{0}^{m} L_{M_{1}}(\Omega), \quad F=W^{-m} E_{\bar{M}_{1}}(\Omega), \quad F_{0}=E_{1}^{*}
$$

\|\|$_{E_{0}}=$ norme induite sur $E_{0}$ par celle de $W_{0}^{m} L_{\mathbb{V}_{1}}(\Omega)$ 


$$
\begin{aligned}
& (,)_{E \times F}=(,)_{W_{0}^{m} L_{M_{1}}(g) \times W^{-m} L_{\bar{M}_{1}}(l)} \\
& (,)_{0}=(,)_{E_{1} \times E_{1}^{*}} \\
& \mathcal{I}_{E_{0}}=\sigma_{0}\left(E_{0}, F\right) \Rightarrow \widehat{I}=\sigma(E, F) \\
& \mathscr{I}_{F_{0}}=\sigma_{0}\left(F_{0}, E_{0}\right) .
\end{aligned}
$$

Associons maintenant aux coefficients $\left\{T_{\alpha}\right\}$ un opérateur $T: W_{0}^{m} L_{N_{1}}(\Omega)$ $\rightarrow W^{-m} L_{\bar{M}_{1}}(\Omega)$ par:

$$
D(T)=\left\{u \in E\left|T_{\alpha}(\xi(u)) \in L_{\bar{M}_{1}}(\Omega), \quad \forall\right| \alpha \mid \leq m\right\}
$$

et

$$
\begin{aligned}
& (T u, v)_{W_{0}^{m} L_{M_{1}}(\theta) \times W^{-m} L_{\bar{M}_{1}}(\Omega)} \\
& \quad=\int_{\Omega} \sum_{|\alpha| \leq m} T_{\alpha}(\xi(u)) D^{\alpha} v d x \quad \forall v \in W_{0}^{m} L_{M_{1}}(\Omega) .
\end{aligned}
$$

On sait (cf [12] Théorème 2) que $T$ est de type pseudomonotone sur tout sous-espace dense de $W_{0}^{m} E_{M_{1}}(\Omega)$ au sens de Gossez [12]. De plus $T$ est fortement quasi-borné sur $E_{0}$ c'est à dire verifie (2) s sur $E_{0}$ (cf la démonstration du Théorème 5.1 de [12]). Ainsi $T$ définit un opérateur $\widetilde{T}$ de $D(\widetilde{T}) \subseteq E \rightarrow F_{0}$ par $\widetilde{T}=p_{1} o T$ où $p_{1}$ désigne l'injection de $W^{-m} L_{M_{1}}(\Omega)$ dans $F_{0}=E_{1}^{*}$ et $\widetilde{T}$ est de type $(G P)_{0}$ est fortement quasiborné sur $E_{0}$, au sens défini ici, puisque $E_{0}$ est dense pour la norme de $E$, dans $W_{0}^{m} E_{M_{1}}(\Omega)$.

On introduit maintenant les hypothèses sur l'opérateur $P$ par (4.1.11). Chaque $P_{\alpha}(x, \eta)$ une fonction définie sur $\Omega \times \boldsymbol{R}^{s_{m-1}}$ à valeurs dans $\boldsymbol{R}$, mesurable sur $x$, pour $\eta$ fixé, et continue suivant $\eta$ pour $x$ fixé.

(4.1.12) $P_{\alpha}(x, \eta)$ est uniformément borné lorsque $\eta$ est borné

(4.1.13) $\quad P_{\alpha}(x, \eta) \eta_{\alpha} \geq 0$ pour presque tout $(x, \eta) \in \Omega \times \boldsymbol{R}^{s_{m-1}}$

On définit alors $P: D(P) \subseteq E F_{0}=E_{1}^{*}$ par

$$
D(P)=\left\{u \in E|| P_{\alpha}(x, \eta(u)) \in L^{1}(\Omega) \text { et } P_{\alpha}(x, \eta(u)) D^{\alpha} u \in L^{1}(\Omega)\right\}
$$

et

$$
(P u, v)_{E_{1^{*} \times E_{1}}}=\int_{\Omega} \sum_{|\alpha| \leq m-1} P_{\alpha}(x, \eta(u)) D^{\alpha} v d x
$$

Lemme 4. 1. 2. L'opérateur $(\widetilde{T}+P)$ est de type $(M)_{0}$ sur $E_{0}$ 
Démonstration. Soit $u_{n} \rightarrow u$ dans $\left(\widehat{E}_{0}, \widehat{\mathcal{I}}\right)$ et $\widetilde{T} u_{n}+P u_{n} \rightarrow f$ dans $\left(F_{0}, \sigma_{0}\left(F_{0}, E_{0}\right)\right)$ avec $f \in F$ et

$$
\lim \sup \left(\widetilde{T} u_{n}+P u_{n}, u_{n}\right)_{0} \leq(f, u) .
$$

Tout d'abord puisque $\widetilde{T}$ est fortement quasi-borné de $E_{0}$ dans $E_{1}{ }^{*}$, on peut supposer que $\widetilde{T} u_{n} \rightarrow g$ dans $\sigma\left(E_{1}{ }^{*}, E_{1}\right)=\sigma_{0}\left(F_{0}, E_{0}\right)$. En fait on a bien plus, on sait que $g \in W^{-m} L_{\bar{M}_{1}}(\Omega)$ et la convergence a lieu suivant $\sigma\left(W^{-m} L_{\bar{M}_{1}}(\Omega), W_{0}^{m} E_{M_{1}}(\Omega)\right)$.

La compacité de l'injection $W_{0}^{m} L_{N_{1}}(\Omega) \hookrightarrow W_{0}^{m-1} L_{M_{1}}(\Omega)$ (cf [12]) entraine en particulier que $D^{\alpha} u_{n}$, pour tout $|\alpha| \leq m-1$ converge p.p. sur 2. Or par (4.1.12), pour chaque $|\alpha| \leq m-1,\left\{P_{\alpha}\left(x, \eta\left(u_{n}\right)\right) D^{\alpha} u_{n}\right\}$ est une suite de fonctions convergentes p.p sur $\Omega$ et positives. Utilisant le lemme de Fatou, comme dans Hess [14], on montre aisément que $u \in D(P)$ et que

$$
\forall|\alpha| \leq m-1 \quad P_{\alpha}\left(x, \eta\left(u_{n}\right)\right) \rightarrow P_{\alpha}(x, \eta(u)) \text { dans } L^{1} \text { fort } .
$$

En particulier grâce à (4.1.13), $P u_{n} \rightarrow P u$ dans $\left(F_{0}, \sigma_{0}\left(F_{0}, E_{0}\right)\right)$, donc $\widetilde{T} u_{n} \rightarrow f-P u$ dans $\left(F_{0}, \sigma_{0}\left(F_{0}, E_{0}\right)\right)$. Or comme $T$ est fortement quasi-borné on peut supposer que $T u_{n} \rightarrow g$ dans $\left(W^{-m} L_{\bar{M}_{1}}(\Omega)\right), \quad \sigma\left(W^{-m} L_{M_{1}}(\Omega)\right.$, $\left.W_{0}^{m} E_{\bar{M}_{1}}(\Omega)\right)$ ). De plus par le lemíne de Fatou

$$
\lim \inf \left(P u_{n}, u_{n}\right)_{0} \geq(P u, u)_{0}
$$

ainsi

$$
\lim \sup \left(\widetilde{T} u_{n}, u_{n}\right)_{0}=\lim \sup \left(T u_{n}, u_{n}\right) \leq(f, u)-(P u, u)_{0} .
$$

Mais $g=f-P u$ ainsi $P u \in W^{-m} L_{U_{1}}(\Omega)$ et par conséquent

$$
\lim \sup \left(T u_{n}, u_{n}\right) \leq(f-P u, u)=(g, u) .
$$

Par le type $(G P)_{0}$ de $T$ il s'ensuit que $T \iota=g$ et donc $f=\widetilde{T} u+P u$.

C.Q.F.D.

Définition 4. 1. 3. Soit $f \in W^{-m} E_{\bar{N}_{1}}(\Omega)$. On dit que $u$ est solution du problème

$$
\widetilde{T} u+P u=f
$$

si $u \in W_{0}^{m} L_{M_{1}}(\Omega)$, et de plus: 


$$
\begin{aligned}
& -T_{\alpha}(x, \xi(u)) \in L_{\bar{M}_{1}}(\Omega) \quad \forall|\alpha| \leq m \\
& -P_{\alpha}(x, \eta(u)) \in L^{1}(\Omega) \text { et } P_{\alpha}(x, \eta(u)) D^{\alpha} u \in L^{1}(\Omega) \quad \forall|\alpha| \leq m-1 \\
& -\int_{\Omega} \sum_{|\alpha| \leq m} T_{\alpha}(x, \xi(u)) D^{\alpha} v d x \\
& \quad+\int_{\Omega|\alpha| \leq m-1} \sum_{\alpha} P_{\alpha}(x, \eta(u)) D^{\alpha} v d x=(f, v) \quad \forall v \in E_{1} .
\end{aligned}
$$

Il découle alors de la Proposition 2.2.1 le

Théorème 4. 1. 4. Pour tout $f \in W^{-m} E_{\bar{M}_{1}}(\Omega)$ il existe une solution du problème (4.1.15).

Démonstration. D'après la Proposition 2.2.1 il suffit de montrer que la condition de coercivité (C3) est realisée avec

$$
\left\{\begin{array}{l}
Y=E=W_{0}^{m} L_{M_{1}}(\Omega) \quad Y_{0}=W_{0}^{m} E_{M_{1}}(\Omega) \\
Z=W^{-m} L_{M_{1}}(\Omega) \quad F=Z_{0}=W^{-m} E_{\bar{M}_{1}}(\Omega) \\
E_{1}=E_{0}, \quad E_{1}^{*}=F_{1}=F_{0} .
\end{array}\right.
$$

Autrement il suffit de montrer que

$$
\lim _{\substack{\|u\|_{\mathbb{E}_{0} \rightarrow+\infty} \\ u \in \boldsymbol{E}_{0}}}(\widetilde{T} u+P u-f, u)_{0}=+\infty .
$$

Or supposons que $(\widetilde{T} u+P u-f, u)_{0} \leq C$, puisque $(P u, u)_{0} \geq 0$ il suit

$$
(T u, u)=(\widetilde{T} u, u)_{0} \leq C+(f, u)_{0} .
$$

On montre alors comme dans Gossez [12], exemple 4.11 que

$$
(f, u)_{0} \leq C_{\varepsilon}+\varepsilon \int_{\Omega} M_{1}\left(D^{\alpha} u\right) d x \text { aù } 0<\varepsilon<d
$$

avec $d$ défini dans (4.1.6).

Grâce à la condition (4.1.7) il s'ensuit alors que $\|u\|_{E_{0}} \leq C_{2}$.

C.Q.F.D.

Remarque. Ce résultat généralise le Théorème 3 de Hess [14] même dans le cas réflexif puisqu'on ne suppose pas $T$ linéaire. De plus la condition de coercivité (C3) est plus faible, dans le cas des opérateurs de type $(M)_{0}$ que celle du Théorème 3 de [14]. 


\section{2. Seconde Application}

Nous allons ici résoudre des problèmes du type envisagés dans l'application précédente. Modifiant certaines hypothèses sur $T$ et $P$, on pourra alors affaiblir les conditions de coercivité et de monotonie.

Les hypothèses sur $T$ sont les suivantes:

$-T$ satisfait à (4.1.3) ainsi que: (cf [13])

(4. 2. 1) Il existe des fonctions $a_{\alpha} \in E_{M_{1}}(\Omega)$ pour $|\alpha|=m$, et à $L_{M_{1}}(\Omega)$ pour $|\alpha|<m$, et des constantes $C_{1}$ et $C_{2}$ telles que pour tout $(x, \xi)$ $\in \Omega \times \boldsymbol{R}^{s_{m}}$

$$
\begin{aligned}
& \text { pour }|\alpha|=m_{1} \quad\left|T_{\alpha}(x, \xi)\right| \leq a_{\alpha}(x)+C_{1} \sum_{|\beta|=m} \bar{M}_{1}^{-1} M_{1}\left(C_{2} \xi_{\beta}\right) \\
& \quad+C_{1} \sum_{|\beta|<m} \bar{M}_{2}^{-1} M_{1}\left(C_{1} \xi_{\beta}\right) \\
& \text { pour }|\alpha|<m \quad\left|T_{\alpha}(x, \xi)\right| \leq a_{\alpha}(x)+C_{1} \sum_{|\beta|=m} \bar{M}_{1}^{-1} M_{2}\left(C_{1} \xi_{\beta}\right) \\
& \quad+C_{1} \sum_{|\beta|<m} \bar{M}_{1}^{-1} M_{1}\left(C_{1} \xi_{\beta}\right)
\end{aligned}
$$

(4.2.2) Il existe un ensemble de fonctions $\left\{b_{\alpha}|| \alpha \mid \leq m\right\}$ telles que $b_{\alpha}$ $\in E_{\overline{\boldsymbol{N}}_{1}}(\Omega)$ si $|\alpha|=m$ et $b_{\alpha} \in L_{\bar{M}_{1}}(\Omega)$ si $|\alpha|<m$, une fonction $b(x) \in L^{1}(x)$ et deux constantes positives $d_{1}$ et $d_{2}$ telles que

$$
\sum_{|\alpha| \leq m}\left(A_{\alpha}(x, \xi)-b_{\alpha}(x)\right) \xi_{\alpha}>d_{1} \sum_{|\alpha| \leq m} M\left(d_{2} \xi_{\alpha}\right)-b(x)
$$

(4. 2. 3) Pour chaque $(x, \eta) \in \Omega \times \boldsymbol{R}^{s_{m-1}}$, $\zeta$ et $\zeta^{\prime} \in R^{s_{m^{\prime}}}$ avec $\zeta \neq \zeta^{\prime}$

$$
\sum_{|\alpha|<m}\left(A_{\alpha}(x, \zeta, \eta)-A_{\alpha}\left(x, \zeta^{\prime}, \eta\right)\right)\left(\zeta_{\alpha}-\zeta_{\alpha}^{\prime}\right)>0
$$

(4. 2. 4) Pour chaque $\left(x, \zeta^{\prime \prime}\right) \in \Omega \times R^{s_{m^{\prime}}}$

$$
\lim _{|\xi| \rightarrow+\infty} \sum_{|\alpha|=m}\left(A_{\alpha}(x, \zeta, \eta)-\zeta_{\alpha}^{\prime}\right)\left(\zeta_{\alpha}-\zeta_{\alpha}^{\prime \prime}\right) \rightarrow+\infty
$$

uniformément pour $\left(\zeta^{\prime}, \eta\right)$ borné dans $\boldsymbol{R}^{s_{m^{1}}} \times \boldsymbol{R}^{s_{m-1}}$.

Les hypothèses sur $P$ sont les suivantes:

$-P$ satisfait à $(4.1 .11),(4.1 .12),(4.1 .13)$ et:

(4.2.5) Pour chaque $\alpha$, tel que $|\alpha| \leq m-1$ et pour chaque $\varepsilon>0$, il existe une constante $K_{\varepsilon \alpha} \geq 0$ telle que

pour tous $\eta_{1}, \eta_{2} \in \boldsymbol{R}^{s_{m-1}}$ 


$$
P_{\alpha}\left(x, \eta_{1}\right) \eta_{2 \alpha} \leq \varepsilon P_{\alpha}\left(x, \eta_{2}\right) \eta_{2 \alpha}+K_{\varepsilon \alpha}\left(1+P_{\alpha}\left(x, \eta_{1}\right) \eta_{1 \alpha}\right)
$$

(on renvoie à Hess [14] [15] pour des exemples de telles fonctions) .

On appelle solution du problème 4.1.15, une solution au sens de la Définition 4.1.3. Les espaces sont ceux de l'application précédente, avec les mêmes conditions sur ces espaces.

On définit maintenant comme dans [13] une famille d'opérateurs $T_{t}$, de $W_{0}^{m} L_{M_{1}}(\Omega) \rightarrow W^{-m} L_{\bar{M}_{1}}(\Omega)$ par

$$
D\left(T_{t}\right)=\left\{u \in W_{0}^{m} L_{M_{1}}(\Omega)\left|T_{\alpha}(x, \xi(u)) \in L_{\bar{M}_{1}}(\Omega), \quad \forall\right| \alpha \mid \leq m\right\}
$$

et

$$
\left(T_{t} u, v\right)_{E \times E^{*}}=\int_{\Omega} \sum_{|\alpha| \leq m}\left(T_{\alpha}(x, \xi(u))-t b_{\alpha}\right) D^{\alpha} v d x \quad \forall v \in W_{0}^{m} L_{M_{1}}(\Omega) .
$$

Choisissant $J$ une application de dualité de $W_{0}^{m} L_{M_{1}}(\Omega) \rightarrow 2^{W_{0}^{-m} L_{M_{1}}(\ell)}$ au sens de Gossez [12] on sait que pour tout $\varepsilon>0$ l'opérateur

$$
T_{\varepsilon, t}=\varepsilon J+T_{t}: E \rightarrow 2^{E^{*}}
$$

constitue une homotopie pseudomonotone bornée au sens de [13].

Definissons $P$ comme dans (4.1.14), et associons a $T_{\varepsilon, t}$ l'application $\widetilde{T}_{\varepsilon, t}: E \rightarrow 2^{F_{0}}$ définie par $\widetilde{T}_{\varepsilon, t}=p o T_{\varepsilon, t}$. On a alors la

Proposition 4. 2. 1. Soit $H_{\varepsilon}=\widetilde{T}_{\varepsilon, t}+P=\widetilde{T}_{t}+P+\varepsilon \widetilde{J}$. Alors $H_{\varepsilon}$ satisfait aux conditions du Corollaire 3.2.3.

Cette proposition est une conséquence immédiate du Théorème 3. 3. 1 compte tenu du fait que l'homotopie "fixe" $P_{t}=P$ satisfait aux hypothèses de ce théorème. Pour une démonstration détaillée de ce fait, elle s'article comme celle du Théorème 5 de Hess [14], et celle de l'application précédente.

Comme conséquence importante on a:

Théorème 4. 2. 2. $\forall f \in W^{-m} E_{\bar{M}_{1}}(\Omega)$, il existe u solution $d u$ problème (4.1.15) (avec les conditions de ce paragraphe).

Remarque. Dans les deux applications précédentes, l'application $p$ était injective. Ce n'est pas le cas en général, lorsque l'on a à traiter 
des problèmes avec des systemes complémentaires quelconques (cf [12]), et des conditions "sur le bord" plus générales.

\section{3. Troisième Application}

Nous allons donner ici, dans un cas très particulier, un autre exemple d'utilisation des résultats du second chapitre. Ce tỷpe de problèmes s'étend à une catégorie plus vaste d'exemples.

Soit $\vec{M}$ une famille de $(n+1)$ fonctions de Young, telles que la famille duale $\vec{N}$ (cf [10]) possède la propriété $\Delta_{2}$, (absolument pas essentiel). On suppose que

$$
\forall|\alpha| \leq 1 \quad M_{\alpha}(t)=\int_{0}^{|t|} P_{\alpha}(t) d t
$$

avec $p$ continue et impaire.

Soit $\left\{\psi_{\beta}\right\}_{|\beta| \leq \kappa}$ une famille de fonctions continues de $\boldsymbol{R} \rightarrow \boldsymbol{R}$ telle que pour tout $|\beta| \leq k, \psi_{\beta}$ soit $(|\beta|+1)$ continument dérivable et que

$$
C \geq \psi_{\beta}^{(|\beta|)}(x) \geq 0 \quad \forall x \in \boldsymbol{R} .
$$

On se propose de résoudre dans un sens convenable l'équation

$$
\sum_{|\alpha| \leq 1}(-1)^{|\alpha|} D^{\alpha}\left(P_{\alpha}\left(D^{\alpha} u\right)\right)+\sum_{|\beta| \leq k}(-1)^{|\beta|} D^{\beta}\left(a_{\beta}(x) D^{\beta}\left(\psi_{\beta}(u)\right)\right)=f
$$

où $\left\{a_{\beta}\right\}$ est une famille de fonctions positives de $L^{\infty}(\Omega)$.

Comme chaque fonction $\psi_{\beta}$ est continument dérivable, $u \rightarrow \psi_{\beta}(u)$ définit un opérateur continu de $L^{2}(\Omega) \rightarrow L^{2}(\Omega)$. Ainsi si l'on désigne par $B$ l'opérateur formel

$$
B u=\sum_{|\beta| \leq k}(-1)^{|\beta|} D^{\beta}\left(a_{\beta}(x) D^{\beta}\left(\psi_{\beta}(u)\right)\right)
$$

$B$ défini un opérateur continu de $L^{2}(\Omega) \rightarrow H^{-2 k}(\Omega)$.

Supposons la famille $\vec{M}$ anisotrope croissante (cf [9]) on sait que l'opérateur $A=\sum_{|\alpha| \leq 1}(-1)^{|\alpha|} D^{\alpha}\left(P_{\alpha}\left(D^{\alpha} u\right)\right)$ constitue un opérateur de $W_{0}^{1} L_{\overrightarrow{\mathrm{k}}}(\Omega) \rightarrow W^{-1} L_{\overrightarrow{\mathrm{N}}}(\Omega)$ de type $(G P)_{0 s}$ sur $W_{0}^{1} E_{\overrightarrow{\mathrm{k}}}(\Omega)$ (pour les topologies faibles définies dans [12]).

Supposons maintenant que

$$
H_{0}{ }^{2 k}(\Omega) \hookrightarrow W_{0}^{1} L_{\vec{M}}(\Omega) \hookrightarrow L^{2}(\Omega) \hookrightarrow W^{-1} L_{\vec{N}}(\Omega) \hookrightarrow H^{-2 k}(\Omega)
$$


avec l'injection de $W_{0}^{1} L_{\vec{M}}(\Omega) \hookrightarrow L^{2}(\Omega)$ compacte. (pour de tels cas cf [7]).

Les opérateurs $A$ et $B$ induisent trivialement des opérateurs $\tilde{A}$ et $\widetilde{B}$ de $W_{0}^{1} L_{\vec{M}}(\Omega) \rightarrow H^{-2 k}(\Omega)$. Choisissant

$$
\begin{aligned}
& E_{0}=H_{0}^{2 k}(\Omega) \quad E=W_{0}^{1} L_{\vec{M}}(\Omega) \quad F=W^{-1} L_{\vec{N}}(\Omega) \quad F_{0}=H^{-2 k}(\Omega) \\
& \|u\|_{\Sigma_{0}}=\|u\|_{W_{0}{ }^{1} \vec{M}_{\vec{M}}^{(\Omega)}} \\
& (u, v)_{0}=(u, v)_{H_{0}{ }^{2 k} \times H^{-2 k}} \text { pour tout }[u, v] \in E_{0} \times F_{0} \\
& (u, v)=(u, v)_{W_{0}{ }^{1} L_{\vec{M}} \times W^{-1} L_{\vec{N}}} \text { pour tout }[u, v] \in E \times F \\
& \mathscr{I}_{E_{0}}=\sigma(E, F)_{E_{0}} \\
& \mathscr{I}_{F_{0}}=\sigma_{0}\left(F_{0}, E_{0}\right)
\end{aligned}
$$

on vérifie aisément que $\widetilde{A}+\widetilde{B}$ est un opérateur de type $(M)_{0}$ sur $\left(E_{0}\right)$ (puisque $B$ est faiblement séquentiellement continu). D'après [12], Lemme 3.14 , et puisque $(B u, u)_{0} \geq 0$, on a la coercivité suivante

$$
\frac{(\tilde{A} u+\widetilde{B} u, u)}{\|u\|_{W_{0}{ }^{1}{ }_{\vec{M}}}} \rightarrow+\infty \text { quand }\|u\|_{w_{0}{ }^{1}{ }_{\vec{M}} \rightarrow+\infty} .
$$

Ainsi de la Proposition 2.2.3 par exemple on obtient le

Théorème 4. 3. 1. Pour tout $f \in W^{-1} L_{\vec{N}}(\Omega)$ il existe $u \in W_{0}^{1} L_{\vec{M}}(\Omega)$ solution de

$$
\tilde{A} u+\widetilde{B} u=f
$$

En particulier $\widetilde{B} u \in W^{-1} L_{\vec{N}}(\Omega)$ et pour tout $v \in H_{0}^{2 k}(\Omega)$

$$
\begin{aligned}
\int_{\Omega} \sum_{|\alpha| \leq 1} P_{\alpha}\left(D^{\alpha} u\right) D^{\alpha} v d x \\
\quad+\sum_{|\beta| \leq k}\left\langle a_{\beta}(x) D^{\beta}\left(\psi_{\beta}(u)\right), D^{\beta} v\right\rangle_{H^{-k}(\Omega) \times H_{0}{ }^{k}(\Omega)} \\
=\langle f, v\rangle_{W^{-1} L_{\vec{N}} \times W_{0}{ }^{1} L_{\vec{N}}} .
\end{aligned}
$$

Remarque. Si les coefficients $\left\{a_{\beta}\right\}$ vérifient certaines conditions, on peut déduire de l'appartenance de $\widetilde{B} u$ à $W^{-1} L_{\vec{N}}(\Omega)$ des conditions de régularité de la solution et simplifier (4.3.4). 


\section{4. Quatrième Application: Equations D’Évolution Paraboliques}

Nous allons illustrer dans cette partie le fait que les méthodes introduites ici, permettent de donner une formulation fonctionnelle à la plupart des équations d'évolution traitées dans Lions [20]. En fait les cas de [20] se traitent avec des espaces réflexifs. Nous allons donner ici un exemple dans le cas non réflexif qui suffira à mettre en évidence les méthodes employées. Cet exemple permettra d'étendre les résultats de Robert [22] qui ne permettent que la résolution d'équation à coefficients rapidement croissants.

Afin de ne pas allonger cet article outre mesure, nous ne traiterons pas le cas le plus général.

Soit $I=[0, T]$ un intervalle fermé borné de $\boldsymbol{R}$. On pose $Q=1 \times \Omega$. Comme dans Robert [21], on note $W_{x}^{m} L_{M}(Q)$ (resp. $W_{x}^{m} E_{M}(Q)$ ) l'espace des fonctions $u$ mesurables telles que pour tout $\alpha$ avec $0 \leq|\alpha| \leq m$ la dérivée partielle $D_{x}^{\alpha} u$ (au sens des distributions) par rapport à la seule variable $x$, appartienne à $L_{M}(Q)$ (resp à $E_{M}(Q)$ ), muni de la norme d'espace de Banach

$$
\|u\|_{W_{x}}=\left(\sum_{0 \leq|\alpha| \leq m}\left\|D_{x}^{\alpha} u\right\|_{L_{M}(Q)}^{2}\right)^{1 / 2} .
$$

On note $\left.W_{0 x}^{m} E_{M}(Q)\right)$ la fermeture pour la norme de $D(Q)$ dans $W_{x}^{m} E_{M}(Q)$ et $W_{0}^{m} L_{M}(Q)$ la fermeture de $D(Q)$ dans $W_{x}^{m} L_{M}(Q)$ pour la topologie $\sigma\left(\pi L_{M}(Q), \pi E_{M}(Q)\right)$. Le dual de $W_{0 x}^{m} E_{M}(Q)$ s'identifie à

$$
W_{x}^{-m} E_{M}(Q)=\left\{f \in \mathscr{D}^{\prime}(Q) \mid f=\sum_{|\alpha| \leq m}(-1)^{|\alpha|} D_{x}^{\alpha} f_{\alpha} \text { avec } f_{\alpha} \in E_{\bar{M}}(Q)\right\}
$$

Remarquons que $\left(W_{0 x}^{m} L_{M}(Q), W_{0 x}^{m} E_{M}(Q), W_{x}^{-m} L_{M}(Q), W_{x}^{-m} E_{M}(Q)\right)$ constitue un système complémentaire (cf [12]).

On choisit maintenant $V$, un espace de Banach reflexif, de dual $V^{\prime}$ tel qu'on ait les injections continues suivantes:

$$
\mathscr{D}(Q) \hookrightarrow V \hookrightarrow L^{2}(Q) \hookrightarrow V^{\prime} \hookrightarrow \mathscr{D}^{\prime}(Q)
$$

On note

$$
W=\left\{u \in \mathscr{D}^{\prime}(Q) \mid u \in V, u^{\prime}=\frac{\partial u}{\partial t} \in V^{\prime}\right\}
$$

muni de la norme 


$$
\|u\|_{W}=\left(\|u\|_{V}^{2}+\left\|u^{\prime}\right\|_{V^{\prime}}^{2}\right)^{1 / 2} .
$$

On supposera dans toute la suite que

$$
W \hookrightarrow \mathscr{C}\left(0, T: L^{2}(\Omega)\right) .
$$

On introduit maintenant un operateur $L: V \rightarrow V^{\prime}$ par

$$
D(L)=\left\{u \in W \mid u(0)=0 \text { et } L u=u^{\prime}=\frac{\partial u}{\partial t} \in V^{\prime}\right\} .
$$

On remarquera que la condition (4.4.1) assure que $L$ est bien défini.

Soit maintenant $J$ un opérateur de dualité de $V \rightarrow V^{\prime}$. On supposera que pour tout $\varepsilon>0,(L+\varepsilon J)$ est de type pseudomonotone (au sens de Brezis [1]).

Remarque. Dans la pratique toutes les hypothèses précédentes seront réalisées, par exemple dans les cas suivants:

- Si $L^{2}(Q) \subseteq L_{M}(Q)$ (ce qui est en général le cas si $M$ possède la propriété $\left.\Delta_{2}\right)$ on choisit $V=L^{2}\left(0, T ; H_{0}{ }^{1}(\Omega)\right)$

-Si $L_{M}(Q) \subseteq L^{2}(Q)$ (ce qui est en général le cas si $\bar{M}$ possède la propriété $A_{2}$ ) on choisit $V=H_{0}^{s}(Q)$ avec $s$ "assez grand".

On suppose ensuite qu'il existe une injection continue de $V \rightarrow W_{0 x}^{m} E_{M}(Q)$. Il est clair que cette injection est à image dense et que sa conjuguée est une injection continue de $W_{x}^{-m} L_{M}(Q) \rightarrow V^{\prime}$. On identifie ainsi $V$ à un sous-espace de $W_{0 x}^{m} L_{M}(Q)$ et $W_{x}^{-m} L_{\bar{M}}(Q)$ à un sous-espace de $V^{\prime}$.

On pose alors

$$
\begin{array}{ll}
E_{0}=V \cap L^{\infty}\left(0, T ; L^{2}(\Omega)\right), & E=W_{0 x}^{m} L_{M}(Q) \cap L^{\infty}\left(0, T ; L^{2}(\Omega)\right) \\
F=W_{x}^{-m} E_{\bar{M}}(Q) & F_{0}=V^{\prime} \\
(v, u)_{0}=(v, u)_{V \wedge V} & \forall[v, u] \in V^{\prime} \times V \\
(v, u)=(v, u)_{W_{0 x}^{m} L_{M}(Q) \times W_{x}^{-m} L_{\bar{M}}(\ell)} \quad \forall[v, u] \in E \times F
\end{array}
$$

$\|u\|_{E_{0}}=$ norme induite par celle de $W_{0 x}^{m} L_{M}(Q)$

$$
\begin{aligned}
& I=\sup \left\{\sigma\left(L^{\infty}\left(0, T ; L^{2}(\Omega)\right), L^{1}\left(0, T ; L^{2}(\Omega)\right)\right), \sigma(E, F)_{{ }_{R_{0}}}\right\} \\
& \mathscr{I}_{F_{0}}=\sigma\left(V^{\prime}, V\right)
\end{aligned}
$$


On définit maintenant un opérateur $A: W_{0 x}^{m} L_{M}(Q) \rightarrow W_{x}^{-m} L_{\bar{M}}(Q)$ de la facon suivante: on considère $\left(A_{\alpha}\right)_{0 \leq|\alpha| \leq m}$ une famille d'application de $Q \times \boldsymbol{R}^{s_{m}} \rightarrow \boldsymbol{R}$ telles que (cf [21]).

(4. 4.2) Pour tout $|\alpha| \leq m, A_{\alpha}(t, x, \xi)$ est une fonction continue de $\xi$ pour $(t, x)$ fixé, et une fonction mesurable de $(t, x)$ pour $\xi$ fixé

(4. 4. 3) Pour tout $|\alpha| \leq m$, il existe $a_{\alpha} \in L^{1}(Q)$ et $b_{\alpha}>0$ tels que pour presque tout $(t, x) \in \mathcal{Q}$ et tout $\xi \in \boldsymbol{R}^{s_{m}}$ on ait:

$$
\bar{M}\left(\left|A_{\alpha}(t, x, \xi)\right|\right) \leq a_{\alpha}(t, x)+b_{\alpha} \sum_{|\beta| \leq m} M\left(\left|\xi_{\beta}\right|\right)
$$

(4. 4. 4) Il existe $\psi \in L^{1}(Q), \psi \geq 0$, et pour tout $\alpha$ avec $0 \leq|\alpha| \leq m$ il existe $g_{\alpha} \in E_{\bar{M}}(Q)$ et $c_{\alpha}>0$ tels que pour presque tout $(t, x) \in Q$ et tout $\xi \in \boldsymbol{R}^{s_{m}}$ on ait:

$$
\sum_{|\alpha| \leq m}\left[A_{\alpha}(t, x, \xi)-g_{\alpha}(t, x)\right] \xi_{\alpha} \geq \sum_{|a| \leq m} C_{\alpha} M\left(\left|\xi_{\alpha}\right|\right)-\psi(t, x)
$$

(4. 4.5) Pour tous $\xi, \xi^{\prime} \in \boldsymbol{R}^{s_{m}}$

$$
\sum_{0 \leq|\alpha| \leq m}\left[A_{\alpha}(t, x, \xi)-A_{\alpha}\left(t, x, \xi^{\prime}\right)\right]\left(\xi_{\alpha}-\xi_{\alpha}^{\prime}\right) \geq 0 .
$$

On définit alors la forme

$$
a(u, v)=\sum_{0 \leq|\alpha| \leq m} \iint_{Q} A_{\alpha}(t, x, \xi(u)) D_{x}^{\alpha} v(t, x) d t d x
$$

et l'opérateur $A$ par

$$
\begin{gathered}
D(A)=\left\{u \in W_{0 x}^{m} L_{M}(Q) \mid a(u, v)\right. \text { est définie et continue } \\
\text { sur } \left.W_{0 x}^{m} E_{M}(Q)\right\}
\end{gathered}
$$

et $(A u, v)_{w_{x}^{-m} L_{\bar{M}^{(}(Q) \times w_{0 x}^{m}}^{m} L_{M}^{(\ell)}}=a(u, v)$.

On sait (cf [21], [12]) que $A$ constitue un opérateur de type pseudomonotone au sens de Gossez de $W_{0 x}^{m} L_{M}(Q) \rightarrow W_{x}^{-m} L_{\bar{M}}(Q)$ pour les topologies du système complémentaire. Il est de plus borné sur $E$. Il induit ainsi un opérateur $\tilde{A}: E \rightarrow V^{\prime}$ de type $(G P)_{0}$ sur $\left(E_{0}\right)$ à notre sens par

$$
\tilde{A}=j^{\prime} A
$$

où $j$ est $1^{\prime}$ injection de $V \rightarrow W_{0 x}^{m} L_{M}(Q)$ et $j^{\prime}$ sa transposée.

Nous allons maintenant résoudre une équation d'évolution associé à l'opérateur $A$, lorsque $L_{M}(Q) \subseteq L^{2}(Q)$ et esquisser sa résolution dans le cas $L^{2}(Q) \subseteq L_{M}(Q)$. 
Supposons donc $L_{M}(Q) \subseteq L^{2}(Q)$. Notant toujours par $\tilde{A}$ la restriction de $\tilde{A}$ à $V$, on pose pour $0 \leq \varepsilon \leq 1$

$$
T_{\varepsilon}=L+\varepsilon J+\tilde{A} \text {. }
$$

$T_{\varepsilon}$ définit un opérateur de $V \rightarrow V^{\prime}$ de type $(M)$ au sens de Brezis [1] et coercif sur $V$ pour $\varepsilon>0$. Ainsi pour tout $\varepsilon>0$

$$
R\left(T_{\varepsilon}\right)=V^{\prime}
$$

On peut alors énoncer le résultat suivant, englobant ceux de Robert [22] en se dispensant d'hypothèses de compatibilité.

Théorème 4. 4. 1. Supposons que pour tout $f \in F$, l'ensemble

$$
\bigcup_{0<\varepsilon \leq 1}\left\{T_{\varepsilon}^{-1}(f)\right\}
$$

reste borné dans $L^{\infty}\left(0, T ; L^{2}(\Omega)\right)$. Alors pour tout $f \in F$, il existe $u \in W_{0 x}^{m} L_{M}(Q) \cap L^{\infty}\left(0, T ; L^{2}(\Omega)\right)$, tel que $A u \in W_{x}^{-m} L_{M}(Q)$, avec

$$
\begin{aligned}
& \frac{\partial u}{\partial t} \in W_{x}^{-m} L_{M}(Q) \text { solution de } \\
& \frac{\partial u}{\partial t}+A u=f
\end{aligned}
$$

Démonstration. Remarquons tout d'abord que l'inclusion de $L_{M}(Q)$ dans $L^{2}(Q)$ nous permet d'affirmer que

$$
(v, u)_{0}=(v, u) \text { pour tout }[u, v] \in E_{0} \times F .
$$

On applique alors le Théorème 3.1.6 à la famille $\left\{T_{\varepsilon}\right\}$. Les hypothèses émises sur $\left\{T_{\varepsilon}\right\}$ ainsi que (4.4.4) joints à la posivité de $L+\varepsilon J$, entrainent que l'ensemble $\underset{0<\varepsilon \leq 1}{\bigcup}\left\{T_{\varepsilon}^{-1}(f)\right\}$ reste relativement compact dans $\left(\widehat{E}_{0}, \widehat{\mathcal{I}}\right)$.

Soit pour tout $\varepsilon>0, u_{\varepsilon}$ tel que

$$
L u_{\varepsilon}+\tilde{A} u_{\varepsilon}+\varepsilon J u_{\varepsilon}=f .
$$

Multipliant (4.4.10) par $u_{\varepsilon}$, il s'ensuit que

$$
\varepsilon\left\|u_{\varepsilon}\right\|_{v}^{2} \leq\left(f, u_{\varepsilon}\right)_{0}-\left(\tilde{A} u_{\varepsilon}, u_{\varepsilon}\right)_{0} \leq C .
$$

Ainsi $\varepsilon J u_{\varepsilon} \rightarrow 0$ dans $V^{\prime}$ fort, et comme l'on peut supposer que $A u_{\varepsilon} \rightarrow g$ dans $W_{x}^{-m} L_{\bar{M}}(Q)$ suivant $\sigma\left(W_{x}^{-m} L_{\bar{M}}(Q), W_{0 x}^{m} E_{M}(Q)\right)$ il s'ensuit que $L u_{\varepsilon}$ 
$\rightarrow f-g$ dans $V^{\prime}$ suivant $\sigma\left(V^{\prime}, V\right)$. Par ailleurs on peut également supposer que $u_{\varepsilon} \rightarrow u$ dans $\left(\widehat{E}_{0}, \widehat{\mathcal{I}}\right)$, donc $L u_{\varepsilon} \rightarrow \frac{\partial u}{\partial t}$ dans $\mathscr{D}^{\prime}(Q)$ et ainsi $\frac{\partial u}{\partial t}=f-g \in W_{x}^{-m} L_{M}(Q)$. Quant au reste de la démonstration, il procède de façon classique. On montre que $u \in D(A)$ et $g=A u$ (pour des détails, cf le cas réflexif Bui An Ton [24]).

C.Q.F.D.

Appliquant ce théorème à $M(u)=e^{|u|}-|u|-1$, ou encore $e^{u^{2}}-1$, ou $u^{2} e^{u^{2}}$, on retrouve les résultats de Robert [21] par d'autres méthodes, qui semblent plus simples.

Dans le cas où $L^{2}(Q) \subseteq L_{M}(Q)$ on peut appliquer des méthodes parfaitement similaires, cependant à ce moment là l'hypothèse (4.4.9) exigée tout au long de ces applications n'est plus réalisée. L'utilisation du Théorème 3.1.6 exige alors quelques petites modifications (toujours réalisées dans la pratique). Soulignons au passage que cette hypothèse (4.4.9) aurait pu être omise tout au long de cet article, mais ceci aurait exigé une présentation nettement plus compliquée.

De plus le Théorème 3.1.6 permet d'énoncer un théorème bien plus général que le Théorème 4.4.1 permettant de résoudre des problèmes du type

$$
\begin{aligned}
& \frac{\partial u}{\partial t}+\sum_{|\alpha| \leq m}(-1)^{|\alpha|} D_{x}^{\alpha} T_{\alpha}(\xi(u(x, t))) \\
& \quad+\sum_{|\alpha| \leq m-1}(-1)^{|\alpha|} D_{x}^{\alpha} P_{\alpha}(\eta(u(x, t)))=f
\end{aligned}
$$

avec des conditions aux limites du type de Dirichlet ou autres, et où les coefficients $\left\{T_{\alpha}\right\}$ et $\left\{P_{\alpha}\right\}$ sont ceux introduits dans les applications 1 et 2. On peut montrer, par des méthodes similaires à celles utilisées dans le Théorème (4.4.1) pour tout $f \in W_{x}^{-m} E_{\bar{M}}(Q)$ l'équation (4.4.11) admet une solution $u \in W_{0 x}^{m} L_{\bar{M}}(Q) \cap L^{\infty}\left(0, T ; L^{2}(\Omega)\right)$ avec $T u \in W_{x}^{-m} L_{\bar{M}}(Q)$ et également pour tout $|\alpha| \leq m-1$

$$
P_{\alpha}(x, \eta(u)) \in L^{1}(Q) \text { et } P_{\alpha}(x, \eta(u)) D_{x}^{\alpha} \in L^{1}(Q) .
$$

Il suffit simplement de procéder à un choix d'espaces et de topologies adéquats. Cette dernière application permet en particulier d'étendre même dans le cas réflexif les résultats de Hess [14] au cas d'équations de 
type parabolique (ou même hyperbolique). Il semble en fait que la résolution du type d'équations considérées dans ces applications soit nouvelle.

Remarques Generales. -Dans toutes les applications précédentes nous avons traité pour plus de simplicité que des problèmes avec conditions aux limites nulles. Des problèmes avec des conditions aux limites plus générales se traitent de façon parfaitement identique.

-Utilisant des résultats du type du Théorème 3.2.2. et les résultats de [28] et [29], on peut résoudre les inéquations variationnelles associées à tous les opérateurs envisagés ci-dessus.

-Dans toutes les applications précédentes, nous nous sommes servis d'un espace $L^{2}(\Omega)$ comme espace pivot. Il est clair des résultats généraux que l'on peut parfaitement se dispenser de ce type d'hypothèse.

-Grâce à [28] et [29], on peut résoudre des problèmes du genre de ceux traités ici mais concernant des opérateurs à domaine plus général, ne contenant pas forcément un sous-espace vectoriel.

Signalons de plus que tous les résultats de cet article, excepté la condition de coercivité du paragraphe 3.2 inspirée de Gossez [12], sont extraits d'une thèse présentée au Technion de Haifa en Juillet 1974.

\section{Références}

[1] Brezis, H., Equations et inéquations non linéaires dans les espaces vectoriels en dualité, Annal. Iknst. Fourier, 18 (1968), 115-175.

[2] Brezis, H., Problèmes unilatéraux, J. Math. Pures et Appl., 51 (1972), 1-168.

[3] Browder, F. E., Existence theory for boundary value problems for quasilinear elliptic systems with strongly nonlinear lower order terms, Proc. Sympos. Pure Math., 23 (1973), 269-286.

[4] Browder, F. E., and Hess, P., Nonlinear Mappings of Monotone type in Banach Spaces, J. Funct. Anal., 11 (1972), 251-294.

[5] Donaldson, T., Inhomogeneous Orlicz-Sobolev spaces and Nonlinear parabolic initial values problems, J. Diff. Eq., 16 (1974), 201-256.

[6] Donaldson, T., Non elliptic boundary value problems in Orlicz-Sobolev spaces, $J$. Diff. Eq., 10 (1971), 507-528.

[7] Donaldson, T., and Trudinger, N., Orlicz-Sobolev spaces and embeddings theorems, $J$. Funct. Anal., 8 (1971), 52-75.

[8] Fitzpatrick, P. M., Surjectivity Results for Nonlinear Mappings from a Banach space to its dual, Math. Annal., 204 (1973), 177-188.

[9] Fougeres, A., Théorèmes de trace et de prolongement dans les espaces de Sobolev et de Sobolev-Orlicz, C. R. Acad. Sc. Paris, Série A, 274 (1972), 181-184. 
[10] Fougeres, A., Opérateurs elliptiques du calcul des variations à coefficients très fortement non linéaires, C. R. Acad. Sci. Paris. Série A, 274 (1972), 763-766.

[11] Gossez, J. P., Opérateurs monotones nonlinéaires dans les espaces de Banach non réflexifs, J. Math. Anal., 34 (1971), 371-395.

[12] Gossez, J. P., Nonlinear elliptic boundary value problems for equations with rapidly or slowly increasing coefficients, Trans. Amer. Math. Soc., 190 (1974), 183-205.

[13] Gossez, J. P., Surjectivity results for pseudomonotone mappings in complementary systems (à paraître).

[14] Hess, J. P., On nonlinear mappings of monotone type, with respect to two Banach spaces, J. Math. Pures Appl., 52 (1973), 13-26.

[15] Hess, P., Variational inequalities for strongly nonlinear elliptic operateurs, J. Math. Pures. Appl., 52 (1973), 285-298.

[16] Hess, P., Théorèmes d'existence pour des perturbations d'opérateurs maximaux monotones, C. R. Acad. Sc. Paris, 275 (1972).

[17] Hess, P., On Nonlinear Mappings of Monotone type Homotopic to odd operators, J. Funct. Anal., 11 (1972), 138-167.

[18] Köthe, G., Topological Vector Spaces, Springer-Verlarg, 1969.

[19] Lacroix, M. T., Espaces de traces des espaces d'Orlicz-Sobolev et applications, $J$. Math. Pures Appl., 9 (1974), 439-458.

[20] Lions, J. L., Quelques méthodes de résolutions des problèmes aux limites non linéaires, DUNOD, 1969.

[21] Robert, J., Inéquations variationnelles paraboliques fortement non linéaires, J. Math. Pures Appl., 53 (1974), 299-321.

[22] Robert, J., Equations d'évolution paraboliques fortement non linéaires, Anal. Scu. Norm. Sup. Pisa, 17 (1975), 247-259.

[23] Ton, B. A., Pseudomonotone operators in Banach Spaces and nonlinear elliptic Equations, Math. Z., 121 (1971), 243-252.

[24] Ton, B. A., On strongly Nonlinear Parabolic Equations, J. Funct. Anal. 7 (1971).

[25] Ma, Tu Wo, Dissertationes Mathematicae XCII.

[26] Wille, F., Monotone Operatoren mit Störungen, Arch. Rat. Mech. Anal., 46 (1972).

[27] Zini, E., A propos de quelques opérateurs non linéaires, Math. Zeit., (1975), 111-138.

[28] Zini, E., Opérateurs de type monotone dans les espaces en dualité (à paraître).

[29] Zini, E., Approximation et surjectivité d'opérateurs de type monotone (à paraître dans Annali di Matematica). 
